\title{
Functional role of Polymerase IV during pollen development in Capsella
}

2 Zhenxing Wang ${ }^{1 *}$, Nicolas Butel ${ }^{1 *}$, Juan Santos-González ${ }^{1}$, Filipe Borges ${ }^{2,3}$, Jun Yi ${ }^{1}$,

3 Robert A. Martienssen ${ }^{2}$, German Martinez ${ }^{1}$, Claudia Köhler ${ }^{1}$

$4 \quad{ }^{1}$ Department of Plant Biology, Swedish University of Agricultural Sciences and Linnean

5 Center for Plant Biology, Uppsala 75007, Sweden

$6 \quad{ }^{2}$ Howard Hughes Medical Institute and Cold Spring Harbor Laboratory, 1 Bungtown

7 Road, Cold Spring Harbor, New York 11724, USA.

${ }^{3}$ Present address: Institut Jean-Pierre Bourgin, INRA, AgroParisTech, Université Paris-

9 Saclay, 78000, Versailles, France

*both authors contributed equally to this work

Short title: Requirement of Polymerase IV in Capsella pollen.

One-sentence summary: Loss of Polymerase IV function in Capsella rubella causes microspore arrest, revealing an important functional role of Polymerase IV during pollen development.

17 The author responsible for distribution of materials integral to the findings presented in this article in accordance with the policy described in the Instructions for Authors 


\section{Abstract}

In Arabidopsis thaliana, the DNA-dependent RNA polymerase IV (Pol IV) is required for the formation of transposable element (TE)-derived small RNA (SRNA) transcripts. These transcripts are processed by DICER-LIKE 3 into 24-nt small interfering RNAs (siRNAs) that guide RNA-dependent DNA methylation. In the pollen grain, Pol IV is also required for the accumulation of 21/22-nt epigenetically-activated siRNAs (easiRNAs) that likely silence TEs by post-transcriptional mechanisms. Despite this proposed functional role, loss of Pol IV function in Arabidopsis does not cause a discernable pollen defect. Here, we show that loss of NRPD1, encoding the largest subunit of Pol IV in the Brassicaceae Capsella rubella, causes post-meiotic arrest of pollen development at the microspore stage. As in Arabidopsis, all TE-derived siRNAs were depleted in Capsella nrpd1 microspores. In wild-type background, we found that the same TEs produced 21/22-nt and 24-nt siRNAs, leading us to propose that Pol IV is generating the direct precursors for 21-24-nt siRNAs, which are targeted by different DICERs. Arrest of Capsella nrpd1 microspores was accompanied by deregulation of genes targeted by Pol IV-dependent siRNAs. The distance of TEs to genes was much closer in Capsella rubella compared to Arabidopsis thaliana, providing a possible explanation for the essential role of Pol IV for pollen development in Capsella. Our study in Capsella uncovers a functional requirement of Pol IV in microspores, emphasizing the relevance of investigating different plant models. 


\section{Introduction}

In flowering plants, male and female gametes are the products of a multistep process that starts from a cell undergoing a meiotic division resulting in haploid spores dividing mitotically to form multicellular gamete-containing gametophytes. The pollen grain corresponds to the male gametophyte and forms after two mitotic divisions of the haploid microspore. The first mitotic division generates the large vegetative cell and a small generative cell that after another mitotic division will give rise to the two sperm cells. The second mitotic division can occur before pollen germination, like in Arabidopsis thaliana, or during pollen germination (Berger and Twell, 2011). Unlike in the male lineage where all microspores will develop into pollen, in most flowering plants only one megaspore survives and mitotically divides to give rise to the seven-celled female gametophyte containing the two female gametes, the egg cell and the central cell (Tekleyohans et al., 2017). In contrast to animals where germ cells separate early from the somatic linage, plant germ cells originate from differentiated cells that acquire the competence to undergo meiotic divisions (Schmidt et al., 2015) The formation of male and female plant gametes is connected with a partial resetting of epigenetic marks that likely serves to achieve meiotic competence (Borges and Martienssen, 2013; Baroux and Autran, 2015; Borg and Berger, 2015). Epigenetic modifications can be applied directly on the DNA in the form of DNA methylation, or on histones, the proteins that package DNA into nucleosomes. The specific type of the modification and its position on the genomic locus defines the transcriptional outcome. DNA methylation is generally (but not always) a repressive modification and is used to silence transposable elements (TEs), but also genes during specific stages of plant development. In plants, DNA methylation can occur in CG, CHG, and $\mathrm{CHH}$ context (where $\mathrm{H}$ correspond to $\mathrm{A}, \mathrm{T}$ or $\mathrm{C}$ ) and is established and maintained by different DNA methyltransferases. Methylation in CG context is maintained by the METHYLTRANSFERASE 1 (MET1), while CHG methylation maintenance requires CHROMOMETHYLASE 3 (CMT3) and to a lesser extent CMT2 (Zhang et al., 2018). The RNA-dependent DNA methylation (RdDM) pathway maintains $\mathrm{CHH}$ methylation by recruiting the DOMAINS REARRANGED METHYLTRANSFERASE 2 (DRM2). This pathway requires the plant-specific DNA-dependent RNA polymerases (Pol) IV and V (Herr et al., 2005; Onodera et al., 2005; Xie et al., 2004). Pol IV generates small 
transcripts of about 30-40-nt in size that are converted into double stranded RNA by the action of the RNA-DEPENDENT RNA POLYMERASE 2 (RDR2) (Blevins et al., 2015; Zhai et al., 2015a; Li et al., 2015). These double stranded RNAs are processed into 23and 24-nt siRNAs by DICER-LIKE 3 (DCL3) (Xie et al., 2004; Singh et al., 2019). The 24nt siRNAs preferentially associate with ARGONAUTE4 (AGO4) and guide DRM2 to its targets by associating with Pol V transcripts (Cao and Jacobsen, 2002; Zilberman et al., 2003; Wierzbicki et al., 2009). Recent studies further uncovered that Pol IV is required for the accumulation of 21/22-nt epigenetically activated siRNAs (easiRNAs) in pollen and establishes a hybridization barrier between plants of different ploidy grades (Martinez et al., 2018; Borges et al., 2018; Satyaki and Gehring, 2019).

Pollen formation in Arabidopsis is connected with reprogramming of $\mathrm{CHH}$ methylation. There is a strong reduction of $\mathrm{CHH}$ methylation during meiosis, which is followed by a restoration of $\mathrm{CHH}$ methylation in the pollen vegetative cell and a locus-specific restoration in sperm (Calarco et al., 2012; Ibarra et al., 2012; Walker et al., 2018). Nevertheless, $\mathrm{CHH}$ methylation is not completely erased during meiosis and locusspecific $\mathrm{CHH}$ methylation was shown to be of functional relevance for meiosis (Walker et al., 2018). In Arabidopsis, the accumulation of meiosis-specific sRNAs depends on Pol IV (Huang et al., 2019) and meiotic defects have been reported in RdDM mutants $r d r 2$, drm2, and ago4, albeit at low frequency in the Columbia (Col) accession (Oliver et al., 2016; Walker et al., 2018). There is furthermore a relaxation of heterochromatin occurring in the vegetative cell as a consequence of histone $\mathrm{H} 1$ depletion, which allows the DNA demethylase DEMETER (DME) to access and demethylate TEs in the vegetative cell (Slotkin et al., 2009; He et al., 2019). Demethylated TEs in the vegetative cell generate siRNAs that can move to sperm cells and may serve to enforce TE silencing in sperm (Martinez et al., 2016; Ibarra et al., 2012; Kim et al., 2019). Nevertheless, the functional relevance of enhanced TE silencing in sperm by mobile siRNAs remains to be demonstrated, since loss of DME function in pollen causes a pollen germination defect, but not a seed defect (Schoft et al., 2011).

Arabidopsis thaliana differs from many other species by its low repeat content of about $24 \%$ of the 135-Mb genome (Maumus and Quesneville, 2014), which may explain its 
112 apparent tolerance to the lack of Pol IV and other RdDM components. Like Arabidopsis

113 thaliana, the closely related Brassicaceae Capsella rubella is a selfer; however, because

114 of its recent transition to selfing (30-100 k years ago (kya)) it has maintained high numbers

115 of TEs and almost half of the $219-\mathrm{Mb}$ genome is repetitive, with many TEs being located

116 in the vicinity to genes (Slotte et al., 2013; Foxe et al., 2009; Guo et al., 2009; Niu et al.,

117 2019). In contrast, Arabidopsis thaliana became a selfer around 500 kya and experienced

118 a strong reduction of TEs (de la Chaux et al., 2012). Given the different TE content in

119 both species, we hypothesized that loss of Pol IV function may have a stronger impact in

120 Capsella rubella. To test this hypothesis, we generated a loss-of-function allele in the

121 Capsella rubella Pol IV subunit NRPD1. We found that loss of NRPD1 in Capsella caused

122 impaired male fertility, as a consequence of a post-meiotic arrest of pollen at the

123 microspore stage. Wild-type microspores accumulated Pol IV-dependent siRNAs in the

124 size range of 21-24-nt, suggesting that the formation of easiRNAs is initiated during or

125 after meiosis. In Capsella and Arabidopsis microspores, 21/22-nt and 24-nt siRNAs were

126 generated from the same TE loci, suggesting that Pol IV is producing the precursors for

127 both types of siRNAs. Consistently, we found that loss of DCL3 in Arabidopsis causes

128 increased formation of 21/22-nt siRNAs, supporting the idea that different DCLs compete

129 for the same double stranded RNA precursor molecule. Microspore arrest in Capsella

130 nrpd1 mutant plants correlated with a substantially stronger deregulation of genes

131 compared to Arabidopsis nrpd1 microspores, including known regulators of pollen

132 development. We conclude that Pol IV in Capsella generates siRNAs in the size range of

133 21-24-nt that have important functional roles for pollen development.

134 Results

135 Loss of Pol IV function causes microspore arrest in Capsella

136 To test the functional role of Pol IV in Capsella rubella, we generated a knockout mutant

137 in the NRPD1 subunit of Pol IV using Crispr/Cas9 (now referred to as Cr nrpd1) (Figure

138 1A). The induced deletion caused the formation of a frameshift and a stop codon after 139385 amino acids, leading to a truncated protein without the catalytically active site 140 (Onodera et al., 2005) that is likely a functional null allele. Like in the Arabidopsis thaliana 141 nrpd1 mutant (referred to as At nrpd1), TE-derived 24-nt siRNAs were abolished in $\mathrm{Cr}$ 
142 nrpd1 leaves (Figure 1B) (Wierzbicki et al., 2012), connected with a strong reduction of $143 \mathrm{CHH}$ methylation levels over TEs (Figure 1C). These results reveal that Pol IV has a 144 conserved function in siRNAs biogenesis and is required for RdDM in Capsella and 145 Arabidopsis.

146 Strikingly, homozygous Cr nrpd1 had strongly reduced seed set (Figure 2A), on average 147 Cr nrpd1 siliques contained only three seeds, corresponding to about $25 \%$ of normal wild148 type seed set. We found that male fertility of Cr nrpd1 was strongly impaired, with most 149 pollen being arrested after meiosis at the microspore stage (Figure 3A-K). Only 150 homozygous Cr nrpd1 mutants were impaired in pollen development, while heterozygous 151 Cr nrpd1 plants were completely fertile and pollen development was normal (Figure 3C, $152 \mathrm{G}, \mathrm{K})$, indicating that loss of Pol IV function affects a stage prior to microspore 153 development, or alternatively, affects tapetum development. Cross-sections of 154 microsporangia confirmed that $\mathrm{Cr}$ nrpd1 mainly formed arrested microspores, with 155 approximate $20 \%$ of the pollen were able to complete development (Figure $3 \mathrm{~L}$ ). There were no obvious differences in tapetum development and degradation in $\mathrm{Cr} n r p d 1$ 157 compared to wild type (Figure 3M-T).

158 Consistent with previous work showing a maternal effect of nrpd1 mutants in Brassica 159 rapa (Grover et al., 2018), about 30\% of ovules of homozygous Cr nrpd1 remained 160 unfertilized after pollination with wild-type pollen, while there was no statistically significant fertility decrease in heterozygous Cr nrpd1 (Figure 2B-E). Inspection of ovules of Cr nrpd1 did not reveal obvious abnormalities; wild-type and Cr nrpd1 ovules contained 163 both an egg cell and unfused polar nuclei at 2 days after emasculation (Figure 2F-H). 164 Furthermore, Cr nrpd1 homozygous, but not heterozygous plants had strongly reduced seed size after self-fertilization or pollination with wild-type pollen (Figure 2I-L), revealing 166 a maternal effect on ovule and seed development.

167 Complementation of Cr nrpd1 with the Arabidopsis NRPD1 genomic sequence under 168 control of the constitutive RPS5A promoter (Weijers et al., 2001) fully restored pollen 169 development in the T1 generation of transgenic plants (Figure 3D,H,L), confirming that 170 the pollen defect is a consequence of impaired Pol IV function and that NRPD1 is 171 functionally conserved in Arabidopsis and Capsella. 
172 Meiotic abnormalities at low frequency were previously reported for mutants of the RdDM

173 pathway in Arabidopsis (Oliver et al., 2016; Walker et al., 2018). However, we did not

174 detect abnormal chromosome segregation during male meiosis in $\mathrm{Cr} n r p d 1$

175 (Supplemental Figure 1) and inspection of meiotic products revealed the formation of

176 tetrads (Supplemental Figure 2), indicating that the pollen arrest after meiosis is not a

177 consequence of a chromosome segregation defect.

\section{Pol IV-dependent silencing of TEs in Capsella microspores}

180 The arrest of Cr nrpd1 pollen at the microspore stage prompted us to compare sRNAs of wild-type and Cr nrpd1 microspores. We enriched for microspores using a percoll gradient

182 following previously established procedures (Dupl'akova et al., 2016). On average, the 183 purity of the fractions was 84\% (Supplemental Figure 3A). Sequencing of isolated sRNAs revealed that TE-derived siRNAs in the size range of 21-24-nt were abolished in $\mathrm{Cr} n r p d 1$ microspores (Figure 4A, Supplemental Figure 4A). Like previously described for Arabidopsis meiocytes (Huang et al., 2019), we observed a strong accumulation of 23-nt siRNAs in Capsella microspores (Figure 4A, Figure 5A). Nearly all TE loci generating 21/22-nt siRNAs also formed 24-nt siRNAs (Figure 4B). To test the functional role of Pol IV-dependent siRNAs in TE silencing, we isolated RNA from wild-type and mutant microspores and performed an RNA-seq analysis. Those TEs that lost 21/22- and 24-nt siRNAs, had higher transcript levels in Cr nrpd1 microspores compared to wild type (Figure 4C), revealing a role of Pol IV-dependent siRNAs in TE silencing in microspores.

193 Together, we conclude that Pol IV-dependent TE-derived siRNAs in the size range of 21-

194 24-nt are present in microspores and are required for TE silencing. This is consistent with 195 recent work proposing that pollen easiRNAs are produced during or shortly after meiosis 196 (Borges et al., 2018).

\section{Pol IV-dependent siRNAs also accumulate in Arabidopsis microspores}

198 Previous work demonstrated that Pol IV generates small transcripts in the size range of 199 30-40-nt that are converted into 24-nt siRNAs by the action of DCL3 (Zhai et al., 2015a; 200 Blevins et al., 2015; Yang et al., 2016). To be able to genetically dissect the Pol IV- 
201 dependent pathway leading to the formation of siRNAs in the size range of 21-24-nt, we

202 tested for the presence of similar siRNAs in Arabidopsis microspores.

203 We sequenced sRNAs from Arabidopsis wild-type and nrpd1 microspores that had been 204 enriched to $90 \%$ following the same procedures as applied for Capsella (Supplemental 205 Figure 3B, Supplemental Figure 4B). Like in Capsella microspores, Arabidopsis 206 microspores accumulated TE-derived siRNAs in the size range of 21-24-nt that were abolished in At nrpd1 microspores (Figure 4D). Thus, 21/22-nt TE-derived siRNAs are already present in microspores and depend on Pol IV, strongly supporting the idea that biogenesis of easiRNAs present in mature pollen starts at an earlier stage, most likely during meiosis. Consistently, microspores and meiocytes as well as microspores and mature pollen grains share a large number of loci generating Pol IV-dependent siRNAs (Figure 5C, D).

To test the functional requirement of Pol IV-derived siRNAs in TE silencing, we correlated the TEs producing Pol IV-dependent 21/22-nt and 24-nt siRNAs to TE expression changes in At nrpd1, lacking Pol IV function. We found a significant association between Pol IV-dependent siRNAs and expression change of TEs in At nrpd1 microspores (Figure 6A) similar to Capsella (Figure 4C), revealing that TE-derived siRNAs are involved in the repression of TE expression in microspores.

Pol IV is usually associated with 24-nt siRNAs through the RdDM pathway and its strong effect on the production of 21/22-nt siRNAs in pollen is thus unexpected. One hypothesis could be that Pol IV transcripts are direct precursors of 21/22-nt siRNAs. If true, we expected that microspore TE-derived 21/22-nt and 24-nt siRNAs should arise from the same genomic loci. Consistently, like in Capsella microspores (Figure 4B), nearly all TE

224 loci generating 21/22-nt siRNAs also formed 24-nt siRNAs (Figure 6B). Visualizing the 225 individual reads in a genome browser showed that all read sizes accumulated along the 226 same loci (Supplemental Figure 5). Taken together, this data show that TE-derived 21/22227 nt siRNAs and 24-nt siRNAs are produced from the same loci in a Pol IV-dependent manner and are able to repress TEs in both Capsella and Arabidopsis. 


\section{TE-derived siRNAs in microspores require RDR2 activity}

231 TE-derived siRNAs were produced from both DNA strands (Figure 6C and Supplemental

232 Figure 5), suggesting the involvement of an RNA-dependent RNA polymerase in the 233 production of the double stranded RNAs used as template for the microspore TE-derived 234 siRNA production.

235 Among the three RDRs with known function in Arabidopsis, RDR2 is tightly associated 236 with Pol IV (Li et al., 2015; Zhai et al., 2015a) and RDR6 has been shown to affect some 237 easiRNAs (Creasey et al., 2014; Martinez et al., 2018). To assess the potential 238 involvement of RDR2 and RDR6 in TE-derived siRNA production in microspores, we 239 analyzed publically available sRNA sequencing data from rdr2 and rdr6 inflorescences 240 (Zhai et al., 2015a; Panda et al., 2016). The sRNA pattern of wild-type and At nrpd1 241 inflorescence tissues was comparable to that of microspores (Figure 4D and Figure 6D), 242 indicating that the siRNAs identified in rdr2 and rdr6 inflorescences are comparable to 243 those of microspores. While rdr6 had no effect on the distribution of TE-derived siRNAs, 244 in rdr2 inflorescences the accumulation of TE-derived siRNAs was abolished (Figure 6D), 245 indicating that RDR2 is likely involved in TE siRNA biogenesis. We also generated siRNA 246 profiles from rdr2 and rdr6 pollen (Figure 5B), which confirm the data obtained from 247 inflorescences and reveal that RDR2, but not RDR6 is required for the generation of 248 21/22-nt siRNAs. These results reinforce the idea that 21/22-nt and 24-nt TE-derived 249 siRNAs present in pollen are processed from a double stranded RNA produced by Pol IV 250 and RDR2.

251 DICERs producing TE-derived siRNAs compete for the same double stranded 252 RNA template

253 Our data suggests that TE-derived siRNAs of different size classes are derived from 254 double stranded RNAs produced by Pol IV/RDR2 (Figure 6B). We hypothesized that the 255 production of different size classes of siRNAs is a consequence of different DICERs 256 competing for the same double stranded RNA template, as it has been previously shown 257 to occur upon disruption of DCL3 function (Gasciolli et al., 2005; Henderson et al., 2006; 258 Kasschau et al., 2007; Bond and Baulcombe, 2015). If true, the impairment of DCL3 
should increase the proportion of Pol IV-dependent 21/22nt siRNAs accumulating over defined loci.

To test this idea, we used publically available sRNA sequencing data of $d c / 3$ inflorescences (Li et al., 2015). We quantified the number of normalized 21/22-nt siRNAs and 24-nt siRNAs mapped against TEs in wild-type and $d c / 3$ inflorescences. In wild type, 24-nt siRNAs were the most abundant siRNA, exceeding the level of 21/22-nt siRNAs by nearly seven fold (Figure $6 \mathrm{E}$ ). In $d c / 3$, the abundance of 21/22-nt siRNAs was highly increased, while 24-nt siRNAs were depleted (Figure 6E). To rule out that the increased abundance of 21/22-nt siRNAs is a consequence of a normalization artifact, we performed the same analysis with miRNAs. As DCL3 is not involved in the 21/22-nt miRNA pathway, observed changes would be suggestive for a normalization artifact. The abundance of 21/22-nt miRNAs was highly similar in wild type and $d c / 3$ (Figure $6 \mathrm{~F}$ ), strongly supporting the notion that the observed increase of 21/22-nt siRNAs in $d c / 3$ inflorescences is not a consequence of a normalization problem. These results indicate that there is indeed a competition between DCL3 and other DCLs for the same double stranded RNA precursor, in agreement with previous data (Gasciolli et al., 2005; Henderson et al., 2006; Kasschau et al., 2007; Bond and Baulcombe, 2015).

\section{TE-derived siRNAs are highly enriched at COPIA95 in Capsella microspores}

To investigate whether Pol IV-dependent siRNAs target similar loci in Arabidopsis and Capsella, we identified sRNA reads mapping to both genomes, which were then mapped to 527 Arabidopsis TE consensus sequences previously reported (Repbase) (Bao et al., 2015). We found a similar number of TE families accumulating Pol IV-dependent siRNAs in Arabidopsis and Capsella microspores, (316 and 301 TE families, respectively), of which 225 were common between both species (Figure 7A). There were substantially fewer TE families forming 24-nt siRNAs in Capsella microspores (133) compared to Arabidopsis (303), but the majority of those overlapped between both species (103). Nearly all TE families forming 24-nt siRNAs also formed 21/22-nt siRNAs (94.1\% (285 out of 303 TE families) in Arabidopsis and 99.2\% (132 out of 133 TE families) in Capsella) (Figure 7A), supporting the idea that 21/22-nt siRNAs and 24-nt siRNAs are derived from the same TE loci in microspores. 
289

290

291

292

293

294

295

296

297

298

299

300

301

302

303

304

305

306

307

308

309

310

311

312

313

314

315

316

317

318

To investigate the specificity of TE-derived siRNAs in Arabidopsis and Capsella microspores, we calculated the proportion of siRNAs targeting specific TE families. Strikingly, we found that nearly $20 \%$ of $21 / 22$-nt siRNAs and more than $40 \%$ of 24 -nt siRNAs were derived from the COPIA95 family (COPIA95 long-terminal repeats (LTR) and internal (I)) in Capsella microspores, while only $0.3 \%$ of both siRNA classes were derived from COPIA95 in Arabidopsis microspores (Figure 7B). We identified 17 and 70 TEs accumulating COPIA95-derived Pol IV-dependent siRNAs in Arabidopsis and Capsella respectively, indicating an expansion of the COPIA95 TE family in Capsella (Supplemental dataset 1). The prominent targeting of COPIA95 in Capsella microspores by Pol IV-dependent siRNAs made us address the question whether loss of Pol IV function may cause increased expression and transposition of COPIA95. Indeed, COPIA95 was highly upregulated in $\mathrm{Cr} n r p d 1$ microspores, but remained silenced in microspores of At nrpd1 (Figure 7C). To test whether increased expression caused heritable transposition, we performed whole genome sequencing of five homozygous $\mathrm{Cr}$ nrpd1 mutants that were derived from homozygous Cr nrpd1 parental plants. We mapped genomic reads to COPIA95 elements and found that one of the five tested mutants had a two-fold increase of COPIA95 elements (Figure 7D). We corroborated this result by using TEPID to identify de novo insertions (Stuart el al 2016) and also detected increased insertions in the same mutant plant (Figure 7D). This result supports the idea that Pol IV is required to prevent TE remobilization in Capsella and is, in particular, required to silence COPIA95.

\section{Loss of Pol IV causes transcriptional changes in microspores}

To understand the cause for post-meiotic arrest of Capsella microspores, we compared the transcriptome changes in $A t$ and $\mathrm{Cr}$ nrpd1 microspores. We found a comparable number of genes being upregulated (log2 fold-change $>1, p<0.05$ ) in $n r p d 1$ microspores of both species; there were however about twice as many genes downregulated (log2 fold-change $<-1, \mathrm{p}<0.05$ ) in Cr nrpd1 microspores compared to At nrpd1 (Supplemental Figure 6A, B; Supplemental dataset 2). While there was no significant overlap between downregulated genes in $\mathrm{Cr}$ and $\mathrm{At}$ nrpd1 microspores (Supplemental Figure 6B), there was a significant overlap of upregulated genes in $\mathrm{Cr}$ and At nrpd1, with a significant 
enrichment for genes with functional roles in stimulus response, cell wall organization, and defense responses (Supplemental Figure 6C).

We tested whether deregulated genes in $\mathrm{Cr}$ and At nrpd1 microspores were targeted by 21/22-nt or 24-nt siRNAs. We found a significant overlap between upregulated genes and downregulated genes with genes losing 21/22-nt and 24-nt siRNAs in Cr nrpd1 (Figure 8A, Supplemental dataset 3). In contrast, in At nrpd1 only downregulated genes significantly overlapped with genes losing 21/22-nt and 24-nt siRNAs (Supplemental Figure 6D, Supplemental dataset 4). Upregulated genes losing 21/22-nt or 24-nt siRNAs in $\mathrm{Cr}$ nrpd1 had functional roles in proteolysis and catabolic processes, cell killing, and interspecies organismal interactions (Figure 8B). The distance of TEs to neighboring genes was significantly shorter in Capsella compared to Arabidopsis, independently of their direction of deregulation (Figure 8C; Wilcoxon test, $p<2 e-15$ ). Nevertheless, upregulated genes in Capsella had an even shorter distance to neighboring TE than nonderegulated or downregulated genes, suggesting an impact of neighboring TEs on gene expression in $\mathrm{Cr}$ nrpd1 microspores. There was however no preference for COPIA95 among those TEs being close to deregulated genes ( $p=1$, hypergeometric test).

Interestingly, we found that downregulated genes associated with loss of 21/22-nt and 24-nt siRNAs in Cr nrpd1 were enriched for genes involved in pollination; among those were known regulators of pollen tube growth like VANGUARD1 (VGD1), ANXUR2 (ANX2), CATION/H+ EXCHANGER 21 (CHX21), and JINGUBANG (JGB) (Figure 8D, Supplemental Figure 7; Jiang et al., 2005; Boisson-Dernier et al., 2005; Lu et al., 2011; Ju et al., 2016). We furthermore identified homologs of pollen receptor kinase encoding genes PRK2 and PRK3 among downregulated genes losing 21/22-nt and 24-nt siRNAs (Figure 8D, Supplemental Figure 7). While there was also a significant overlap of downregulated genes in At nrpd1 with genes losing siRNAs (Supplemental Figure 6D), those genes were not enriched for genes involved in pollination (Supplemental Figure 6E) and the aforementioned genes were not deregulated in At nrpd1 (Figure 8D). The affected pollen receptor kinases have partly redundant functions in pollen tube growth and perception of female attractant peptides (Chang et al., 2013; Takeuchi and Higashiyama, 2016). Importantly, RNAi-mediated knockdown of PiPRK1, a PRK homologue Petunia, 
causes microspore arrest (Lee et al., 1996), suggesting that reduced expression of PRKs may contribute to the $\mathrm{Cr} n r p d 1$ microspore arrest. All genes were highly induced in the microspores to mature pollen transition (Figure $8 \mathrm{E}$ ), suggesting that their expression is required to ensure viable pollen formation, a hypothesis that remains to be tested.

\section{Discussion}

In this manuscript, we report that loss of Pol IV function in Capsella rubella causes arrest of microspore development and a maternal effect on ovule and seed development, strongly differing from the lack of obvious reproductive abnormalities of $n r p d 1$ mutants in Arabidopsis (Mosher et al., 2009). Previous work revealed that mutations in NRPD1, NRPE1 and RDR2 in Brassica rapa cause a maternal effect on seed development, while no defect in pollen development was reported (Grover et al., 2018). The mutation in $B$. rapa NRPD1 was not a null allele; however, the mutation in $R D R 2$ completely abolished production of 24-nt siRNAs (Grover et al., 2018), indicating that this mutant was a functional null for RDR2. Since loss of Cr NRPD1 caused a similar molecular effect as mutations in Arabidopsis and B. rapa NRPD1 (depletion of 24-nt siRNAs and $\mathrm{CHH}$ methylation) (Wierzbicki et al., 2012; Panda et al., 2016; Grover et al., 2018) and that the Cr nrpd1 mutant could be complemented with the Arabidopsis NRPD1 sequence, we conclude that the molecular function of Pol IV is likely conserved between the three species, but the targets differ. Interestingly, loss of Pol IV function in tomato also causes sterility, but the cause for this phenotype remains to be explored (Gouil and Baulcombe, 2016). The microspore arrest in Cr nrpd1 is possibly a consequence of TEs being in close vicinity to either essential regulators of microspore development, like PRKs, or genes that cause microspore arrest upon overexpression. The distance of TEs to neighboring genes is substantially larger in Arabidopsis compared to Capsella, supporting this notion.

We observed a heritable remobilization of the COPIA95 element in progenies of $\mathrm{Cr}$ nrpd1 mutant, consistent with this element being preferentially targeted by Pol IV-generated siRNAs in Capsella and strongly activated in Cr nrpd1 microspores. Interestingly, in Arabidopsis, the COPIA element ONSEN also undergoes transgenerational 
retrotransposition in nrpd1 after heat treatment and new ONSEN insertions differ between siblings derived from a single plant (Ito et al., 2011). Since amplification of COPIA 95 was only observed in one of the five tested $\mathrm{Cr}$ nrpd1 progenies, it seems unlikely that the consistently observed microspore arrest is connected to TE remobilization. Alternatively, it is possible that only those microspores survive where TE remobilization did not occur, or did occur at low frequency.

Cr nrpd1 microspores were completely depleted for TE-derived siRNAs, including 21/22nt siRNAs, which are usually not associated with Pol IV (Xie et al., 2004; Zhai et al., 2015a; Blevins et al., 2015). A similar depletion of 21/22-nt siRNAs (easiRNAs) was previously reported in the mature pollen grain of At nrpd1 mutants (Martinez et al., 2018; Borges et al., 2018). The biogenesis of easiRNAs was suggested to be a consequence of reduced heterochromatin formation in the vegetative cell and resulting TE activation (Slotkin et al., 2009; Creasey et al., 2014). Based on genetic data Borges et al. (2018) proposed that easiRNA biogenesis occurs earlier, during or early after meiosis. Our data reveal that 21/22-nt TE-derived siRNAs are already present in the microspores and given the similarity to meiocyte siRNAs (Huang et al., 2019), they are likely generated before or during meiosis.

In rice and maize, highly abundant 21-nt phasiRNAs accumulate in premeiotic anthers and 24-nt phasiRNAs are enriched in meiotic-stage anthers (Zhai et al., 2015b; Johnson et al., 2009; Komiya et al., 2014). The 21-nt phasiRNAs were shown to be important for male fertility in rice and disruption of 24-nt phasiRNA production yields conditional male sterility in maize (Fan et al., 2016; Teng et al., 2018). Biogenesis of premeiotic and meiotic phasiRNAs in maize seems to occur in the tapetum, rather than in meiocytes where they accumulate (Zhai et al., 2015b). The production of 24-nt phasiRNAs depends on miR2275, a pathway that is widely present in the eudicots, but missing in the Brassicaceae (Xia et al., 2019), suggesting evolutionary divergence of the functional role of phasiRNAs during pollen development. Cross-sections did not reveal obvious tapetal defects in Cr nrpd1, indicating that microspore arrest in Cr nrpd1 is not a consequence of a tapetal defect. 
407 The strong dependency of TE-derived siRNA accumulation on Pol IV suggests that Pol 408 IV transcripts are the precursors for all sizes of TE-derived siRNAs in microspores. 409 Previous work revealed that in the absence of DCL3, other DCL proteins (DCL1, DCL2, 410 and DCL4) are able to process Pol IV transcripts into 21- or 22-nt siRNAs (Gasciolli et al., 411 2005; Henderson et al., 2006; Kasschau et al., 2007; Bond and Baulcombe, 2015). We 412 thus propose that before or during meiosis, Pol IV transcripts are targeted by other DCLs 413 than only DCL3, explaining why all sizes of Pol IV-dependent siRNAs derive from the 414 same TE loci.

415 In Arabidopsis siliques, a nuclear localized form of DCL4 was shown to target Pol IV 416 transcripts and generates 21-nt siRNAs (Pumplin et al., 2016). The abundance of those 417 21-nt Pol IV-derived siRNAs was nevertheless low, contrasting to the high abundance in 418 microspores. One possible explanation could be that the disruption of the nuclear 419 envelope during meiosis allows cytoplasmic DCLs to gain access to Pol IV/RDR2 420 transcripts. This implicates that meiosis is the trigger of Pol IV-dependent 21-24-nt siRNA 421 production, consistent with our genetic data. Not mutually exclusive with this scenario is 422 the possibility that 22-nt siRNAs produced during meiosis trigger secondary 21/22-nt 423 siRNA production in the mature pollen grain by targeting TEs transcripts expressed in the 424 vegetative cell of pollen (Slotkin et al., 2009). This amplification of the signal by the 425 canonical post-transcriptional gene silencing (PTGS) pathway (Martinez de Alba et al., 426 2013) should result in high abundant 21/22-nt siRNAs in mature pollen, which is in 427 agreement with published siRNA profiles of pollen (Martinez et al., 2018; Borges et al., 428 2018).

429 We have shown that Pol IV-dependent 21/22-nt siRNAs are required to silence TEs in 430 microspores. This could be achieved by the non-canonical RdDM pathway involving 431 21/22-nt siRNAs (Cuerda-Gil and Slotkin, 2016); or, alternatively, by the PTGS pathway. 432 Levels of $\mathrm{CHH}$ methylation are low in meiocytes, but increase in microspores and in the 433 vegetative cell of pollen (Walker et al., 2018). Nevertheless, CHH methylation in 434 microspores is very low (Calarco et al., 2012), making it more likely that TE silencing in 435 microspores and later on in the vegetative cell is achieved by PTGS, consistent with the 436 high accumulation of 21/22-nt siRNAs in mature pollen. 
Recent work from our and other groups revealed that disruption of NRPD1 suppresses the hybridization barrier between plants of different ploidy grades (Martinez et al., 2018; Satyaki and Gehring, 2019). However, while Martinez et al. (2018) did not find a suppressive effect when using mutants in RdDM components such as RDR2 and NRPE1, Satyaki and Gehring (2019) found those mutants to suppress hybrid seed failure. The difference between both studies lies in the use of tetraploid RdDM mutants by Satyaki and Gehring (2019), while RdDM mutants introgressed into omission of second division 1 (osd1) were used by Martinez et al. (2018). Loss of OSD1 suppresses the second meiotic division, leading to unreduced gamete formation (d'Erfurth et al., 2009). Here, we showed that RDR2 is required for easiRNA biogenesis, suggesting that loss of easiRNAs is not sufficient to suppress the triploid block induced by the osd1 mutation. An important difference between osd1 and tetraploid plants is the ploidy of the genome at the beginning of the meiosis, which is diploid and tetraploid, respectively. This fact can have a strong impact, since tetraploid plants undergo DNA methylation changes leading to stable epialleles (Mittelsten Scheid et al., 2003). Given that DNA methylation recruits Pol IV (Law et al., 2013; Zhang et al., 2013) and our study points that easiRNAs are generated during meiosis, it is possible that the requirement of RdDM activity for easiRNA formation and ploidy barriers may be different depending of the initial ploidy of the plants. If true, the signal establishing the triploid block depends on Pol IV but only indirectly on RdDM, suggesting both pathways can be separated, as previously proposed in maize endosperm (Erhard Jr. et al., 2013).

In summary, our study in Capsella uncovers a functional requirement of Pol IV in microspores, emphasizing that Pol IV-dependent siRNA formation occurs earlier than previously hypothesized (Slotkin et al., 2009). We show that Pol IV is generating the precursors for 21-24-nt siRNAs, which may be a consequence of different DCLs being able to access Pol IV transcripts during meiosis. Our study highlights the relevance of 463 investigating different plant models to gain novel insights into the molecular control of developmental processes 


\section{Methods}

\section{Plant growth and material}

Mutants alleles nrpd1-3 (Salk_128428) and dc/3-1 (Salk_005512) have been previously described (Pontier et al., 2005; Xie et al., 2004). For all experiments using Arabidopsis thaliana, the Col-0 accession was used as wild type, while for Capsella rubella, accession Cr1GR1 was used.

Seeds of Arabidopsis and Capsella were surface sterilized in 5\% commercial bleach and $0.01 \%$ Tween-20 for $10 \mathrm{~min}$ and washed three times in sterile ddH2O. Seeds were sown on $1 / 2$ MS-medium $(0.43 \%$ MS-salts, $0.8 \%$ Bacto Agar, $0.19 \%$ MES hydrate and $1 \%$ Sucrose). After stratification for 2 days at $4^{\circ} \mathrm{C}$, plates were transferred to a growth chamber (16 h light / $8 \mathrm{~h}$ dark; $110 \mu \mathrm{mol} / \mathrm{s} / \mathrm{m} 2 ; 21^{\circ} \mathrm{C} ; 70 \%$ humidity). After 10 days, seedlings were transferred to soil and grown in a growth chamber (16 h light / $8 \mathrm{~h}$ dark; $110 \mu \mathrm{mol} / \mathrm{s} / \mathrm{m} 2 ; 21^{\circ} \mathrm{C} ; 70 \%$ humidity). Capsella plants were grown in the growth chamber at the same light-dark cycles, but at $18{ }^{\circ} \mathrm{C}$ and $60 \%$ humidity.

\section{Generation of plasmids and transgenic plants}

The web tool CRISPR-P (http://cbi.hzau.edu.cn/cgi-bin/CRISPR) was used to design the sgRNAs for knocking out Capsella NRPD1 (Carubv10019657m) (Lei et al., 2014). Sequence information for the primers containing the two sgRNA sequences are listed in 484 Supplemental table 1. They were used for amplifying the fragment including Target1sgRNA-scaffold-U6-terminator-U6-29promoter-Target2 using plasmid DT1T2-PCR as

486 template (Wang et al., 2015). The amplified fragment was digested with Bsal and inserted 487 into pHEE401E containing an egg cell specific promoter driven Cas9 cassette as previous 488 described (Wang et al., 2015).

489 The pHEE401E-NRPD1-T1T2 construct was transformed into Agrobacterium 490 tumefaciens (GV3101) and bacteria containing the plasmid were used to transform 491 Capsella rubella accession Cr1GR1 by floral dip (Clough and Bent, 1998). The genomic 492 sequence of Arabidopsis NRPD1 with the stop codon was amplified from Col genomic 493 DNA and cloned into pDONR221 (Invitrogen) and after being confirmed by sequencing it 
494

495

496

497

498

499

500

501

502

503

504

505

506

507

508

509

510

511

512

513

514

515

516

517

518

519

520

was inserted in pB7WG2 in which the CaMV35S promoter was replaced by the 1.6-kb promoter sequence of RPS5A (Weijers et al., 2001).

\section{Microscopy}

Capsella inflorescences were harvested and fixed in 3:1 (Ethanol : acetic acid) solution. Pollen were manually dissected from stage 12 and 13 anthers, and then stained with DAPI (1 $\mu \mathrm{g} / \mathrm{ml})$ as previous described (Brownfield et al., 2015). The slides were observed using a Zeiss Axio Scope.A1 and a Zeiss 7800 confocal microscope.

To generate sections, Capsella inflorescences were harvested and fixed in FAA solution (50\% Ethanol, 5\% acetic acid, 4\% formaldehyde) and embedded using the Leica Historesin Embedding Kit (702218500). Three-micrometer sections were prepared using a HM 355 S microtome (Microm) with glass knives. Sections were stained with $0.1 \%$ toluidine blue for $1 \mathrm{~min}$, washed five times with distilled water, air dried and then observed using Zeiss Axio Scope.

\section{Microspore extraction}

The different pollen stages were extracted on a percoll gradient following previously published procedures (Dupl'akova et al., 2016). The purity of each fraction was assessed by Alexander and DAPI staining.

\section{RNA and small RNA sequencing}

RNA of Arabidopsis microspores was isolated using the TRIzoL following the manufacturer's protocol (Thermofischer: cat-15596018). Purified RNA was treated with DNAsel (Thermofischer: cat-EN0521) and then loaded on a 15\% TBE-Urea polyacrylamide gel. RNA with a size of 15-27-nt was retrieved and eluted by crushing the gel in PAGE elution buffer (1M Tris pH7.5, 2.5M NaOAc, 0.5M EDTA pH8) followed by an overnight incubation and a new TRizoL extraction.

Capsella leaves were ground with liquid nitrogen, and $100 \mathrm{mg}$ of fine powder from each sample was used for RNA isolation. Capsella microspores were ground in a pre-cooled mortar with Lysis/Binding Solution of the mirVana ${ }^{\text {TM }}$ miRNA isolation kit. Both long RNAs 
521 (>200-nt) and short RNAs (<200-nt) were isolated from leaves and microspores according

522 to the manufacturer's protocol (mirVana ${ }^{\text {TM }}$ miRNA Isolation Kit, AM1560). Size selection

523 of sRNAs was performed as described above.

524 For the RNA seq analysis, total RNA was treated with the Poly(A) mRNA Magnetic

525 Isolation Module kit (NEB \#E7490). Libraries were prepared from the resulting mRNA with

526 the NEBNext巴 Ultra ${ }^{\mathrm{TM}}$ II kit (NEB \#E7770S). sRNA seq libraries were generated with the

527 NEBNext巴 Multiplex Small RNA kit (NEB \#E7300S). RNA-seq libraries and sRNA-seq

528 libraries were sequenced at the SciLife Laboratory (Uppsala, Sweden) and Novogene

529 (Hongkong, China) on a HiSeqX in 150-bp paired-end mode or a Illumina HiSeq2000 in

530 50-bp single-end mode, respectively.

\section{Bisulfite sequencing}

532 Leaves of 6-10 plants of Capsella wildtype and nrpd1 mutants were pooled as one 533 replicate. Genomic DNA was extracted using the MagJET Plant Genomic DNA Kit 534 (K2761). The bisulfite conversion and library preparation were done as previously 535 described (Moreno-Romero et al., 2016). Libraries were sequenced at the SciLife 536 Laboratory (Uppsala, Sweden) on an Illumina HiSeq2000 in 125-bp paired-end mode.

\section{DNA sequencing}

538 Genomic DNA was isolated from leaves of one Capsella wild-type plant and five pol iv 539 mutants using the MagJET Plant Genomic DNA Kit (K2761). Libraries were generated 540 using the NEBNext ${ }^{\circledR}$ Ultra ${ }^{\mathrm{TM}}$ II DNA Library Prep Kit for Illumina $\circledR$ and sequenced at 541 Novogene (Hongkong, China) on an Illumina HiSeqX in 150-bp paired-end mode.

\section{Bioinformatic analysis}

543 For sRNA data, adapters were removed from the 50-bp long single-end sRNA reads in 544 each library. The resulting 18-30-bp long reads were mapped to the respective reference 545 genomes using bowtie (-v 0 --best). All reads mapping to chloroplast and mitochondria 546 and to structural noncoding RNAs (tRNAs, snRNAs, rRNAs, or snoRNAs) were removed. 547 Mapped reads from both replicates were pooled together, sorted in two categories (21/22- 
548

549

550

551

552

553

554

555

556

557

558

559

560

561

562

563

564

565

566

567

568

569

570

571

572

573

574

575

576

577

578

nt and 24-nt long) and remapped to the same reference masked genome mentioned above using ShortStack (--mismatches 0 --mmap f) (Johnson et al., 2016) in order to improve the localization of sRNAs mapping to multiple genomic locations. We normalized the alignments by converting coverage values to RPM values. TE-siRNAs were defined as siRNAs that overlap with annotated TEs. TEs accumulating 20 or more reads in the merged wild-type libraries were considered as TE producing siRNA loci. TEs losing siRNAs in nrpd1 were defined as those having less than $5 \%$ of reads left in $n r p d 1$ compared to wild-type samples. To identify genes losing siRNAs in nrpd1 microspores, we determined siRNA coverage over the genomic loci plus $2 \mathrm{~kb}$ up-and downstream regions and calculated differences to wild-type microspores using the Bioconductor RankProd Package (Hong et al 2006) (log2 fold change <-1, (percentage of false prediction) pfp<0.05). For RNA analysis, for each replicate, reads were mapped to the Arabidopsis and the Capsella reference genomes, using TopHat v2.1.0 (Trapnell et al, 2009 ) in single-end mode. Gene and TE expression was normalized to reads per kilobase per million mapped reads (RPKM) using GFOLD (Feng et al, 2012). For Capsella the $C$. rubella v1.0 annotated genome was used as reference (Slotte et al., 2013, https://phytozome.jgi.doe.gov/pz/portal.html\#!info?alias=Org_Crubella), which was also used as reference in all Capsella analyses describe herein. For Arabidopsis the TAIR10 annotation was used. Expression level for each condition was calculated using the mean of the expression values in both replicates. Differentially regulated genes and TEs across the two replicates were detected using the rank product method, as implemented in the Bioconductor RankProd Package (Hong et al 2006). For DNA methylation analysis, reads of each pair were split in 50-bp-long fragments and mapped in single-end mode using Bismark (Krueger and Andrews, 2011). Duplicated reads (aligning to the same genomic position) were eliminated and methylation levels for each condition were calculated averaging the replicates.

To estimate the number and identity of sRNA reads mapping to COPIA95 TEs in Capsella, 21/22-nt and 24-nt sRNA reads were first mapped to a consensus reference fasta file for Arabidopsis TEs available at Repbase (https://www.girinst.org/repbase/update/index.html) (Jurka et al 2005) using bowtie (-v 2 -m 3 --best --strata). Reads mapping to COPIA95 TEs were remapped to the C. rubella 
579 reference genome with ShortStack (-mismatches 0 -mmap f) (Johnson et al., 2016) and

580 normalized using coverage values of single copy genes.

581 New TE insertions in Capsella rubella were identified using TEPID (Stuart et al., 2016) in

582 pair-end mode based on the sequenced genomes of five $\mathrm{Cr}$ pol iv mutants and the 583 corresponding wild-type.

\section{Data availability}

585 The sequencing data generated in this study are available in the Gene Expression 586 Omnibus under accession number GSE129744. Supplemental Table 2 summarizes all 587 sequencing data generated in this study.

\section{Author Contributions and Acknowledgments}

590 ZW, NB, and CK performed the experimental design. ZW, NB, JY, and FB performed experiments. GM advised on experimental work. RAM contributed experimental data. ZW, 592 NB, JSG, and CK analyzed the data. ZW, NB, JSG, and CK wrote the manuscript. All 593 authors read and commented on the manuscript.

594 We are grateful to Cecilia Wärdig for technical assistance. Sequencing was performed by 595 the SNP\&SEQ Technology Platform, Science for Life Laboratory at Uppsala University, 596 a national infrastructure supported by the Swedish Research Council (VRRFI) and the 597 Knut and Alice Wallenberg Foundation. This research was supported by grants from the 598 Swedish Research Council VR and Formas (to CK), a grant from the Knut and Alice 599 Wallenberg Foundation (to CK), and support from the Göran Gustafsson Foundation for 600 Research in Natural Sciences and Medicine (to CK). Research in the Martienssen 601 laboratory is supported by the US National Institutes of Health (NIH) grant R01 602 GM067014, and by the Howard Hughes Medical Institute. The authors acknowledge 603 assistance from the Cold Spring Harbor Laboratory Shared Resources, which are funded 604 in part by the Cancer Center Support Grant (5PP30CA045508).

\section{References}


Bao, W., Kojima, K.K., and Kohany, O. (2015). Repbase Update, a database of repetitive elements in eukaryotic genomes. Mob DNA 6: 11.

Baroux, C. and Autran, D. (2015). Chromatin dynamics during cellular differentiation in the female reproductive lineage of flowering plants. Plant $\mathrm{J} 83: 160-176$.

Berger, F. and Twell, D. (2011). Germline specification and function in plants. Annu Rev Plant Biol 62: 461-484.

Blevins, T., Podicheti, R., Mishra, V., Marasco, M., Wang, J., Rusch, D., Tang, H., and Pikaard, C.S. (2015). Identification of Pol IV and RDR2-dependent precursors of 24 nt siRNAs guiding de novo DNA methylation in Arabidopsis. Elife 4: e09591.

Boisson-Dernier, A., Roy, S., Kritsas, K., Grobei, M.A., Jaciubek, M., Schroeder, J.I., Grossniklaus, U. (2009) Disruption of the pollen-expressed FERONIA homologs ANXUR1 and ANXUR2 triggers pollen tube discharge. Development 136: 32793288.

Bond, D.M. and Baulcombe, D.C. (2015). Epigenetic transitions leading to heritable, RNA-mediated de novo silencing in Arabidopsis thaliana. Proc Natl Acad Sci U S A 112: 917-922.

Borg, M. and Berger, F. (2015). Chromatin remodelling during male gametophyte development. Plant J 83: 177-188.

Borges, F. and Martienssen, R.A. (2013). Establishing epigenetic variation during genome reprogramming. RNA Biol 10: 490-494.

Borges, F., Parent, J.S., van Ex, F., Wolff, P., Martinez, G., Kohler, C., and Martienssen, R.A. (2018). Transposon-derived small RNAs triggered by miR845 mediate genome dosage response in Arabidopsis. Nat Genet 50: 186-192.

Brownfield, L., Yi, J., Jiang, H., Minina, E.A., Twell, D., and Köhler, C. (2015). Organelles maintain spindle position in plant meiosis. Nat. Commun.

Calarco, J.P., Borges, F., Donoghue, M.T., Van Ex, F., Jullien, P.E., Lopes, T., Gardner, R., Berger, F., Feijo, J.A., Becker, J.D., and Martienssen, R.A. (2012). Reprogramming of DNA methylation in pollen guides epigenetic inheritance via small RNA. Cell 151: 194-205.

Cao, X. and Jacobsen, S.E. (2002). Role of the arabidopsis DRM methyltransferases in de novo DNA methylation and gene silencing. Curr Biol 12: 1138-1144. 
Chang, F., Gu, Y., Ma, H., Yang, Z. (2013). AtPRK2 promotes ROP1 activation via RopGEFs in the control of polarized pollen tube growth. Mol Plant 6: 1187-1201. Clough, S.J. and Bent, A.F. (1998). Floral dip: a simplified method for Agrobacteriummediated transformation of Arabidopsis thaliana. Plant J 16: 735-743.

Creasey, K.M., Zhai, J., Borges, F., Van Ex, F., Regulski, M., Meyers, B.C., and Martienssen, R.A. (2014). miRNAs trigger widespread epigenetically activated siRNAs from transposons in Arabidopsis. Nature 508: 411-415.

Cuerda-Gil, D. and Slotkin, R.K. (2016). Corrigendum: Non-canonical RNA-directed DNA methylation. Nat Plants 3: 16211.

d'Erfurth, I., Jolivet, S., Froger, N., Catrice, O., Novatchkova, M., and Mercier, R. (2009). Turning Meiosis into Mitosis. Plos Biol. 7.

Dupl'akova, N., Dobrev, P.I., Renak, D., and Honys, D. (2016). Rapid separation of Arabidopsis male gametophyte developmental stages using a Percoll gradient. Nat Protoc 11: 1817-1832.

Erhard Jr., K.F., Parkinson, S.E., Gross, S.M., Barbour, J.E., Lim, J.P., and Hollick, J.B. (2013). Maize RNA polymerase IV defines trans-generational epigenetic variation. Plant Cell 25: 808-819.

Fan, Y. et al. (2016). PMS1T, producing phased small-interfering RNAs, regulates photoperiod-sensitive male sterility in rice. Proc Natl Acad Sci U S A 113: 1514415149.

Feng J., Meyer C.A., Wang Q., Liu J.S., Shirley Liu X., and Zhang Y. (2012) GFOLD: a generalized fold change for ranking differentially expressed genes from RNA-seq data. Bioinformatics 28: 2782-2788.

Foxe, J.P., Slotte, T., Stahl, E.A., Neuffer, B., Hurka, H., and Wright, S.I. (2009). Recent speciation associated with the evolution of selfing in Capsella. Proc Natl Acad Sci $U$ S A 106: 5241-5245.

Gasciolli, V., Mallory, A.C., Bartel, D.P., Vaucheret, H. (2005) Partially redundant functions of Arabidopsis DICER-like enzymes and a role for DCL4 in producing trans-acting siRNAs. Curr Biol 15: 1494-1500.

Grover, J.W., Kendall, T., Baten, A., Burgess, D., Freeling, M., King, G.J., and Mosher, R.A. (2018). Maternal components of RNA-directed DNA methylation are required 
for seed development in Brassica rapa. Plant J 94:575-582.

Gouil, Q., Baulcombe, D.C. (2016) DNA Methylation Signatures of the Plant Chromomethyltransferases. PLoS Genet 12: e1006526.

Guo, Y.L., Bechsgaard, J.S., Slotte, T., Neuffer, B., Lascoux, M., Weigel, D., and Schierup, M.H. (2009). Recent speciation of Capsella rubella from Capsella grandiflora, associated with loss of self-incompatibility and an extreme bottleneck. Proc Natl Acad Sci U S A 106: 5246-5251.

He, S., Vickers, M., Zhang, J., and Feng, X. (2019). Natural depletion of histone H1 in sex cells causes DNA demethylation, heterochromatin decondensation and transposon activation. Elife 8.

Henderson, I.R., Zhang, X., Lu, C., Johnson, L., Meyers, B.C., Green, P.J., and Jacobsen, S.E. (2006). Dissecting Arabidopsis thaliana DICER function in small RNA processing, gene silencing and DNA methylation patterning. Nat Genet 38: 721-725.

Herr, A.J., Jensen, M.B., Dalmay, T., and Baulcombe, D.C. (2005). RNA polymerase IV directs silencing of endogenous DNA. Science 308: 118-120.

Hong, F., Breitling, R., McEntee, C.W., Wittner, B.S., Nemhauser, J.L., and Chory, J. (2006). RankProd: a bioconductor package for detecting differentially expressed genes in meta-analysis. Bioinformatics 22: 2825-2827.

Huang, J., Wang, C., Wang, H., Lu, P., Zheng, B., Ma, H., Copenhaver, G.P., and Wang, Y. (2019). Meiocyte-specific and AtSPO11-1-dependent Small RNAs and Their Association with Meiotic Gene Expression and Recombination. Plant Cell. Ibarra, C.A. et al. (2012). Active DNA demethylation in plant companion cells reinforces transposon methylation in gametes. Science 337: 1360-1364.

Ito H, Gaubert H, Bucher E, Mirouze M, Vaillant I, Paszkowski J. (2011) An siRNA pathway prevents transgenerational retrotransposition in plants subjected to stress. Nature 472: 115-119.

Jiang, L., Yang, S.L., Xie, L.F., Puah, C.S., Zhang, X.Q., Yang, W.C., Sundaresan, V., and Ye, D. (2005). VANGUARD1 encodes a pectin methylesterase that enhances pollen tube growth in the Arabidopsis style and transmitting tract. Plant Cell 17: 584-596. 
699

700

701

702

703

704

705

706

707

708

709

710

711

712

713

714

715

716

717

718

719

720

721

722

723

724

725

726

727

728

729

Johnson, C., Kasprzewska, A., Tennessen, K., Fernandes, J., Nan, G.L., Walbot, V., Sundaresan, V., Vance, V., and Bowman, L.H. (2009). Clusters and superclusters of phased small RNAs in the developing inflorescence of rice. Genome Res 19: 1429-1440.

Johnson, N.R., Yeoh, J.M., Coruh, C., and Axtell, M.J. (2016). Improved Placement of Multi-mapping Small RNAs. G3 6: 2103-2111.

Ju, Y., Guo, L., Cai, Q., Ma, F., Zhu, Q.Y., Zhang, Q., and Sodmergen (2016). Arabidopsis JINGUBANG Is a Negative Regulator of Pollen Germination That Prevents Pollination in Moist Environments. Plant Cell 28: 2131-2146.

Kasschau, K.D., Fahlgren, N., Chapman, E.J., Sullivan, C.M., Cumbie, J.S., Givan, S.A., and Carrington, J.C. (2007). Genome-wide profiling and analysis of Arabidopsis siRNAs. PLoS Biol 5: e57.

Kim, M.Y., Ono, A., Scholten, S., Kinoshita, T., Zilberman, D., Okamoto, T., and Fischer, R.L. (2019). DNA demethylation by ROS1a in rice vegetative cells promotes methylation in sperm. Proc Natl Acad Sci U S A.

Komiya, R., Ohyanagi, H., Niihama, M., Watanabe, T., Nakano, M., Kurata, N., and Nonomura, K. (2014). Rice germline-specific Argonaute MEL1 protein binds to phasiRNAs generated from more than 700 lincRNAs. Plant J 78: 385-397.

Krueger, F. and Andrews, S.R. (2011). Bismark: A flexible aligner and methylation caller for Bisulfite-Seq applications. Bioinformatics.

de la Chaux, N., Tsuchimatsu, T., Shimizu, K.K., and Wagner, A. (2012). The predominantly selfing plant Arabidopsis thaliana experienced a recent reduction in transposable element abundance compared to its outcrossing relative Arabidopsis lyrata. Mob DNA 3: 2.

Law, J.A., Du, J., Hale, C.J., Feng, S., Krajewski, K., Palanca, A.M., Strahl, B.D., Patel, D.J., and Jacobsen, S.E. (2013). Polymerase IV occupancy at RNA-directed DNA methylation sites requires SHH1. Nature 498: 385-389.

Lee H, Karunanandaa B, McCubbin A, Gilroy S, Kao T (1996). PRK1, a receptor-like kinase of Petunia inflata, is essential for postmeiotic development of pollen. Plant $\mathrm{J}$ 9: 613-624.

Lei, Y., Lu, L., Liu, H.Y., Li, S., Xing, F., and Chen, L.L. (2014). CRISPR-P: a web tool 
for synthetic single-guide RNA design of CRISPR-system in plants. Mol Plant 7: 1494-1496.

Lei, M., Zhang, H., Julian, R., Tang, K., Xie, S., Zhu, J.K. (2015) Regulatory link between DNA methylation and active demethylation in Arabidopsis. Proc Natl Acad Sci U S A 112: 3553-3557.

Li, S., Vandivier, L.E., Tu, B., Gao, L., Won, S.Y., Li, S., Zheng, B., Gregory, B.D., and Chen, X. (2015). Detection of Pol IV/RDR2-dependent transcripts at the genomic scale in Arabidopsis reveals features and regulation of siRNA biogenesis. Genome Res 25: 235-245.

Lu, Y., Chanroj, S., Zulkifli, L., Johnson, M.A., Uozumi, N., Cheung, A., and Sze, H. (2011). Pollen tubes lacking a pair of $\mathrm{K}+$ transporters fail to target ovules in Arabidopsis. Plant Cell 23: 81-93.

Martinez de Alba, A.E., Elvira-Matelot, E., and Vaucheret, H. (2013). Gene silencing in plants: a diversity of pathways. Biochim Biophys Acta 1829: 1300-1308.

Martinez, G. et al. (2018). Paternal easiRNAs regulate parental genome dosage in Arabidopsis. Nat Genet 50: 193-198.

Martinez, G., Panda, K., Kohler, C., and Slotkin, R.K. (2016). Silencing in sperm cells is directed by RNA movement from the surrounding nurse cell. Nat Plants 2: 16030.

Maumus, F. and Quesneville, H. (2014). Ancestral repeats have shaped epigenome and genome composition for millions of years in Arabidopsis thaliana. Nat Commun 5: 4104.

Mittelsten Scheid, O., Afsar, K., and Paszkowski, J. (2003). Formation of stable epialleles and their paramutation-like interaction in tetraploid Arabidopsis thaliana. Nat. Genet.

Moreno-Romero, J., Jiang, H., Santos-Gonzalez, J., and Kohler, C. (2016). Parental epigenetic asymmetry of PRC2-mediated histone modifications in the Arabidopsis endosperm. EMBO J 35: 1298-1311.

Mosher, R.A., Melnyk, C.W., Kelly, K.A., Dunn, R.M., Studholme, D.J., and Baulcombe, D.C. (2009). Uniparental expression of PollV-dependent siRNAs in developing endosperm of Arabidopsis. Nature 460: 283-286.

Niu, X.M., Xu, Y.C., Li, Z.W., Bian, Y.T., Hou, X.H., Chen, J.F., Zou, Y.P., Jiang, J., Wu, 
Q., Ge, S., Balasubramanian, S., and Guo, Y.L. (2019). Transposable elements drive rapid phenotypic variation in Capsella rubella. Proc Natl Acad Sci U S A 116: 6908-6913.

Oliver, C., Santos, J.L., and Pradillo, M. (2016). Accurate chromosome segregation at first meiotic division requires ago4, a protein involved in RNA-dependent DNA methylation in Arabidopsis thaliana. Genetics 204: 543-553.

Onodera, Y., Haag, J.R., Ream, T., Costa Nunes, P., Pontes, O., and Pikaard, C.S. (2005). Plant nuclear RNA polymerase IV mediates siRNA and DNA methylationdependent heterochromatin formation. Cell 120: 613-622.

Panda, K., Ji, L., Neumann, D.A., Daron, J., Schmitz, R.J., and Slotkin, R.K. (2016). Full-length autonomous transposable elements are preferentially targeted by expression-dependent forms of RNA-directed DNA methylation. Genome Biol 17: 170.

Pontier, D., Yahubyan, G., Vega, D., Bulski, A., Saez-Vasquez, J., Hakimi, M.A., LerbsMache, S., Colot, V., and Lagrange, T. (2005). Reinforcement of silencing at transposons and highly repeated sequences requires the concerted action of two distinct RNA polymerases IV in Arabidopsis. Genes Dev 19: 2030-2040.

Pumplin, N., Sarazin, A., Jullien, P.E., Bologna, N.G., Oberlin, S., and Voinnet, O. (2016). DNA methylation influences the expression of DICER-LIKE4 isoforms, which encode proteins of alternative localization and function. Plant Cell 28: 27862804.

Satyaki, P.R. V and Gehring, M. (2019). Paternally acting canonical RNA-directed DNA methylation pathway genes sensitize Arabidopsis endosperm to paternal genome dosage. Plant Cell 31: 1563-1578.

Schmidt, A., Schmid, M.W., and Grossniklaus, U. (2015). Plant germline formation: common concepts and developmental flexibility in sexual and asexual reproduction. Development 142: 229-241.

Schoft, V.K., Chumak, N., Choi, Y., Hannon, M., Garcia-Aguilar, M., Machlicova, A., Slusarz, L., Mosiolek, M., Park, J.S., Park, G.T., Fischer, R.L., and Tamaru, H. (2011). Function of the DEMETER DNA glycosylase in the Arabidopsis thaliana male gametophyte. Proc Natl Acad Sci U S A 108: 8042-8047. 
Singh, J., Mishra, V., Wang, F., Huang, H.Y., and Pikaard, C.S. (2019). Reaction Mechanisms of Pol IV, RDR2, and DCL3 Drive RNA Channeling in the siRNADirected DNA Methylation Pathway. Mol Cell 75: 576-589.

Slotkin, R.K., Vaughn, M., Borges, F., Tanurdzic, M., Becker, J.D., Feijo, J.A., and Martienssen, R.A. (2009). Epigenetic reprogramming and small RNA silencing of transposable elements in pollen. Cell 136: 461-472.

Slotte, T. et al. (2013). The Capsella rubella genome and the genomic consequences of rapid mating system evolution. Nat Genet 45: 831-835.

Stuart, T., Eichten, S.R., Cahn, J., Karpievitch, Y. V, Borevitz, J.O., and Lister, R. (2016). Population scale mapping of transposable element diversity reveals links to gene regulation and epigenomic variation. Elife 5: e20777.

Takeuchi, H., Higashiyama, T. (2016). Tip-localized receptors control pollen tube growth and LURE sensing in Arabidopsis. Nature 531: 245-248.

Tekleyohans, D.G., Nakel, T., and Gross-Hardt, R. (2017). Patterning the Female Gametophyte of Flowering Plants. Plant Physiol 173: 122-129.

Teng, C., Zhang, H., Hammond, R., Huang, K., Meyers, B.C., and Walbot, V. (2018). Dicer-like 5 deficiency confers temperature-sensitive male sterility in maize. bioRxiv.

Trapnell, C., Pachter, L., and Salzberg, S.L. (2009). TopHat: discovering splice junctions with RNA-Seq. Bioinformatics 25: 1105-1111.

Walker, J., Gao, H., Zhang, J., Aldridge, B., Vickers, M., Higgins, J.D., and Feng, X. (2018). Sexual-lineage-specific DNA methylation regulates meiosis in Arabidopsis. Nat Genet 50: 130-137.

Wang, Z.P., Xing, H.L., Dong, L., Zhang, H.Y., Han, C.Y., Wang, X.C., and Chen, Q.J. (2015). Egg cell-specific promoter-controlled CRISPR/Cas9 efficiently generates homozygous mutants for multiple target genes in Arabidopsis in a single generation. Genome Biol 16: 144.

Weijers, D., Geldner, N., Offringa, R., and Jurgens, G. (2001). Seed development: Early paternal gene activity in Arabidopsis. Nature 414: 709-710.

Wierzbicki, A.T., Cocklin, R., Mayampurath, A., Lister, R., Rowley, M.J., Gregory, B.D., Ecker, J.R., Tang, H., and Pikaard, C.S. (2012). Spatial and functional relationships among Pol V-associated loci, Pol IV-dependent siRNAs, and cytosine methylation 
in the Arabidopsis epigenome. Genes Dev 26: 1825-1836.

Wierzbicki, A.T., Ream, T.S., Haag, J.R., and Pikaard, C.S. (2009). RNA polymerase V transcription guides ARGONAUTE4 to chromatin. Nat Genet 41: 630-634.

Villar, C., Erilova, A., Makarevich, G., Trösch, R., Köhler, C. (2009) Control of PHERES1 imprinting in Arabidopsis by direct tandem repeats. Mol Plant 2: 654660.

Xia, R., Chen, C., Pokhrel, S., Ma, W., Huang, K., Patel, P., Wang, F., Xu, J., Liu, Z., Li, J., and Meyers, B.C. (2019). 24-nt reproductive phasiRNAs are broadly present in angiosperms. Nat Commun 10: 627.

Xie, Z., Johansen, L.K., Gustafson, A.M., Kasschau, K.D., Lellis, A.D., Zilberman, D., Jacobsen, S.E., and Carrington, J.C. (2004). Genetic and functional diversification of small RNA pathways in plants. PLoS Biol 2: E104.

Xu, H., Knox, R.B., Taylor, P.E., and Singh, M.B. (1995). Bcp1, a gene required for male fertility in Arabidopsis. Proc Natl Acad Sci U S A 92: 2106-2110.

Yang, D.L., Zhang, G., Tang, K., Li, J., Yang, L., Huang, H., Zhang, H., and Zhu, J.K. (2016). Dicer-independent RNA-directed DNA methylation in Arabidopsis. Cell Res 26: 1264.

Zhai, J. et al. (2015a). A one precursor one siRNA model for Pol IV-dependent siRNA biogenesis. Cell 163: 445-455.

Zhai, J., Zhang, H., Arikit, S., Huang, K., Nan, G.L., Walbot, V., and Meyers, B.C. (2015b). Spatiotemporally dynamic, cell-type-dependent premeiotic and meiotic phasiRNAs in maize anthers. Proc Natl Acad Sci U S A 112: 3146-3151.

Zhang, H. et al. (2013). DTF1 is a core component of RNA-directed DNA methylation and may assist in the recruitment of Pol IV. Proc Natl Acad Sci U S A 110: 82908295.

Zhang, H., Lang, Z., and Zhu, J.K. (2018). Dynamics and function of DNA methylation in plants. Nat Rev Mol Cell Biol 19: 489-506.

Zhang, Y., McCormick, S. (2007). A distinct mechanism regulating a pollen-specific guanine nucleotide exchange factor for the small GTPase Rop in Arabidopsis thaliana. Proc Natl Acad Sci U S A 104: 18830-18835.

Zilberman, D., Cao, X., and Jacobsen, S.E. (2003). ARGONAUTE4 control of locus- 

specific siRNA accumulation and DNA and histone methylation. Science (80-. ). 299: 716-719.

\section{Figure legends}

\section{Figure 1. Disruption of NRPD1 in Capsella impairs 24-nt siRNA formation and} RdDM.

(A) Deleted genomic region in Capsella NRPD1 at 1634 - 2108 bp (genomic sequence).

Target 1 (T1) and target (T2) sequences of Crispr/Cas9 are indicated.

(B) Profile of TE-derived siRNAs in Capsella wild-type (wt) and nrpd1 leaves.

(C) DNA methylation levels at TEs in Capsella wt and nrpd1 leaves.

\section{Figure 2. Loss of NRPD1 in Capsella affects female fertility and causes a reduction} of seed size.

(A) Total seed numbers per silique in wild-type (wt) (11 siliques) and Cr nrpd1 mutant (24 siliques) plants. ${ }^{* * *} p<0.001$ (Student's t-test). (B-D) Siliques at 2 days after pollination (DAP) from (B) wt $\times$ wt, (C) nrpd1/+ $\times$ wt, (D) $n r p d 1 \times$ wt crosses. Bar (B-D): $1 \mathrm{~mm}$. Asterisks mark unfertilized ovules. (E) Fertilization frequency in indicated crosses. 16 siliques per cross combination were analyzed. ${ }^{* *} \mathrm{p}<0.01$ (Student's t-test). (F-H) Ovules at 2 days after emasculation of $(F) w t,(G) n r p d 1 /+,(H) n r p d 1$ plants. Bar $(F-H): 20 \mu m$. $(\mathrm{I}-\mathrm{K})$ Seeds harvested from (I) wt $\times \mathrm{wt},(\mathrm{J}) n r p d 1 /+\times \mathrm{wt},(\mathrm{K}) n r p d 1 \times \mathrm{wt}$ crosses. Bar $(\mathrm{I}-\mathrm{K})$ : $1 \mathrm{~mm}$. (L) Seed size of mature seeds derived from wt $\times$ wt $(n=120), n r p d 1 /+\times$ wt $(n=149)$, $n r p d 1 \times$ wt $(n=64)^{* * *} p<0.001$ (Student's t-test). n.s, not significant.

\section{Figure 3. Cr nrpd1 pollen arrest at the microspore stage.}

(A-D) Bright field and (E-H) corresponding DAPI staining of manually dissected pollen from anthers at stage 12/13. Pollen of wt ( $A$ and $E$ ), Cr nrpd1 homozygotes ( $B$ and F), $\mathrm{Cr}$ nrpd1 heterozygotes ( $C$ and $G$ ), and a complemented line (D and $H)$. Bar (A-H): $50 \mu \mathrm{m}$. Confocal images of DAPI stained pollen of wild type (wt) (I), Cr nrpd1 homozygotes (J), and $\mathrm{Cr} n r p d 1$ heterozygotes (K). Bar (I-K): $5 \mu \mathrm{m}$. (L) Percentage of mature pollen (MP) in anthers dissected at stage 12/13 from wt, Cr nrpd1 homozygotes (nrpd1), Cr nrpd1 heterozygotes (nrpd1/+) and a complemented line (compl.). Numbers of pollen counted 
885

886

887

888

889

890

891

892

893

894

895

896

897

898

899

900

901

902

903

904

905

906

907

908

909

910

911

912

913

914

915

in each genotype were shown on top of the bars. Microsporangia cross-sections stained with Toluidine Blue at anther stage 8 ( $\mathrm{M}$ and $\mathrm{O}), 11(\mathrm{Q}$ and $\mathrm{S})$, and 12 ( $\mathrm{R}$ and $\mathrm{T})$ of wt (M, $\mathrm{Q}$ and $\mathrm{R})$ and $\operatorname{Cr} \operatorname{nrod1}(\mathrm{O}, \mathrm{S}$ and $\mathrm{T})$. Bar (M,O,Q-T): $50 \mu \mathrm{m}$. Insets in (M) and (O) are shown enlarged in $(N)(P)$, respectively. Bar (N and $P): 50 \mu m$. wt, wild type.

Figure 4. Pol IV is required for 21-24-nt siRNAs in Capsella microspores.

(A) Profile of TE- derived siRNAs in Capsella wt and nrpd1 microspores. (B) Abundance of TE-derived Cr NRPD1-dependent 21-22-nt siRNAs and 24-nt siRNAs in Capsella microspores. Values are indicated as the log10 of the average RPM of both libraries. Each dot represents one TE for a total of 5455 TEs. The correlation has been tested by a Spearman test (correlation coefficient 0.6872). (C) Loss of 21/22-nt and 24-nt siRNAs at TEs associates with increased transcript level of TEs in $\mathrm{Cr} n r p d 1$ microspores. Increasing accumulation of siRNAs over TEs is plotted from low to high levels of accumulation. Only TEs with siRNAs more in wt than in $\mathrm{Cr} n r p d 1$ are represented. Differences between first and last categories are significant $(P=3.4 \mathrm{e}-13$ and $1.4 \mathrm{e}-9$, respectively, Wilcoxon test). (D) sRNA profile of TE-derived siRNAs from Arabidopsis wt and nrpd1 microspores.

\section{Figure 5. Meiocytes, microspores and mature pollen grain accumulate overlapping} sets of siRNAs.

(A) TE-derived siRNA distribution in Arabidopsis meiocytes of the indicated genetic background (data from Huang et al., 2019). (B) TE-derived siRNA distribution in Arabidopsis pollen grains of the indicated genetic background. (C) Upset plot showing the overlap of TEs accumulating 21/22-nt siRNAs or 24-nt siRNAs in Arabidopsis microspores and meiocytes (data from Huang et al., 2019). (D) Upset plot showing the overlap of TEs accumulating 21/22-nt siRNAs or 24-nt siRNAs in Arabidopsis microspores and mature pollen grain (MPG) (data from Martinez et al., 2018).

\section{Figure 6. Pol IVIRDR2 generate templates for 21-24-nt siRNAs.}

(A) Loss of Pol IV-dependent 21/22-nt easiRNAs associates with increased transcript levels of TEs in Arabidopsis microspores. Increasing accumulation of siRNAs over TEs is 
916 plotted from low to high levels of accumulation. In both plots, siRNAs levels at TEs in wt

917 increase from left to right in quantiles. Differences between first and last categories are 918 significant ( $p=2.6 \mathrm{e}-10$ and $1.5 \mathrm{e}-14$, respectively, Wilcoxon test). (B) Abundance of At 919 NRPD1-dependent 21-22-nt siRNAs and 24-nt siRNAs at TEs in Arabidopsis microspores.

920 Values are indicated as log10 of the average reads per million (RPM) of both libraries. 921 Each dot represents one TE for a total of 1504 TEs. The correlation has been tested by 922 a Spearman test (correlation coefficient 0.7686). (C) Plots showing the distribution of the 923 ratio of the number of reads mapped against the positive strand to the total number of 924 mapped reads. Left plots shows analysis for 21/22-nt reads, right plot for 24-nt reads. (D)

925 TE-derived siRNA distribution in inflorescences of rdr2 (left panel; data from Zhai et al., 926 2015) and rdr6 (right panel; data from Panda et al., 2016).

927 (E) Average total 21/22-nt or 24-nt reads mapping against TEs in wt or dc/3 libraries (data 928 from Li et al., 2015). Reads were normalized to show RPM values. (F) Average total 929 21/22-nt reads mapping against miRNAs in wt or dc/3 libraries (data from Li et al., 2015).

930 Reads were normalized to show RPM values.

931

Figure 7. COPIA95-siRNAs are highly enriched in Capsella microspores.

933 (A) Upset plots of TE families accumulating Pol IV-dependent 21/22-nt and 24-nt siRNAs 934 in Arabidopsis (At) and Capsella (Cr). (B) Proportions of Pol IV-dependent 21/22-nt and 935 24-nt siRNAs accumulating at specific TE consensus sequences in relation to all TE936 siRNAs. Reads mapping to COPIA95 long-terminal repeats (LTR) and internal (I) 937 sequences are highlighted in red and yellow, respectively. (C) Log2 expression fold 938 change of mRNAs for COPIA95 elements in nrpd1 mutant microspores of Arabidopsis 939 and Capsella compared to the corresponding wild type. ${ }^{* *} p<0.01$ (Student's t-test). (D) 940 Relative number of COPIA95 insertions (left panel) and total TE insertions (right panel) 941 compared to the corresponding wild-type control in five progenies of homozygous $\mathrm{Cr}$ 942 nrpd1.

943

944 Figure 8. Deregulated genes differ in Arabidopsis and Capsella nrpd1 mutant 945 microspores. 
(A) Venn diagrams showing overlap of deregulated genes ( $\mid \log _{2}$ fold change $\mid>1, p<0.05$ ) in nrpd 1 microspores of Capsella and genes losing 21/22-nt and 24-nt siRNAs at 2kb upand downstream and gene body ( $\log _{2}$ fold change $\left.<-1, p<0.05\right)$ in Capsella nrpd1 microspores. (B) Enriched gene ontologies (GOs) for biological processes of intersected genes losing siRNAs and deregulated genes in Capsella nrpd1 microspores. Top 5 GOs of each analysis are shown. (C) Distance of Arabidopsis and Capsella genes to closest TEs. All: all genes, up: significantly upregulated genes, down: significantly downregulated genes. ${ }^{*} \mathrm{p}<0.05,{ }^{* *} \mathrm{p}<0.01,{ }^{* *} \mathrm{p}<0.001$, n.s, not significant. (Statistical analysis: Wilcoxon test). (D) $\log _{2}$ expression fold change of VGD1, PRK2, PRK3, CHX21, JGB and $A N X 2$ genes in nrpd1 microspores compared to wild type (wt) in Capsella (Cr) and Arabidopsis (At). (E) mRNA levels of VGD1, PRK2, PRK3, CHX21, JGB and ANX2 in Arabidopsis wild-type meiocytes, microspores and mature pollen grain (MPG). We added plus 1 to all values to avoid negative $\log _{10}$ values.

\section{Supplemental Figure legends}

Supplemental Figure 1. Meiosis is not affected in Capsella nrpd1. Supports Figure 2.

Meiosis in Capsella wild-type (A - H) and nrpd1 ( - P) plants. A and I, pachytene. B and J, diakinesis. C and K, metaphase I. D and L, telophase I. E and M, prophase II. F and $\mathrm{N}$, metaphase II. G and O, anaphase II. H and P, telophase II. Bar: $5 \mu \mathrm{m}$.

\section{Supplemental Figure 2. Normal tetrad formation in Capsella wild type (A) and pol} iv (B). Supports Figure 2.

Shown are whole mount confocal images. Bar: $20 \mu \mathrm{m}$.

\section{Supplemental Figure 3. Average purity of Capsella and Arabidopsis microspore} extractions. Supports Figure 4.

Microspore extractions of Capsella (A) and Arabidopsis (B) were tested by DAPI staining and the $\mathrm{B} 2$ and $\mathrm{B} 1$ fractions were selected as the fractions containing the highest proportion of microspores in Capsella and Arabidopsis, respectively. Shown is 
976 the average percentage of four and eight independent extractions in Capsella and

977 Arabidopsis, respectively. Error bars show standard deviation.

Supplemental Figure 4. Profile of total sRNAs in Capsella (A) and Arabidopsis (B) microspores. Supports Figure 4.

981

Supplemental Figure 5. Example of four loci producing Pol IV-dependent siRNAs in Arabidopsis. Supports Figure 7.

984 Bars represent normalized reads. The color indicates the length of the analyzed reads: red 24-nt, blue 22-nt, and green 21-nt. The DNA strand is indicated by the (+) or (-). TE sequences are represented in yellow. microspores. Supports Figure 8.

(A) Venn diagram showing overlap of upregulated genes in nrpd1 microspores of Venn diagram showing overlap of downregulated genes in nrpd1 microspores of Capsella and Arabidopsis. Significance was determined by a hypergeometric test. (C) Enriched gene ontologies (GOs) for biological processes of upregulated genes shared in Arabidopsis and Capsella nrpd1 microspores. Top 5 GOs are shown. (D) Venn diagrams showing overlap of deregulated genes ( $\mid \log _{2}$ fold change| $>1, p<0.05$ ) in $n r p d 1$ microspores of Arabidopsis and genes losing 21/22-nt and 24-nt siRNAs at 2kb up-and downstream and gene body ( $\log _{2}$ fold change < $-1, p<0.05$ ) in Arabidopsis nrpd1 microspores. Significance was determined by a hypergeometric test. 21/22-nt and 24-nt siRNAs in Capsella microspores.

\section{Supplemental datasets}

1004 Supplemental dataset 1. COPIA95 elements accumulating Pol IV-dependent 1005 siRNAs in Capsella and Arabidopsis. 
1006 Supplemental dataset 2. Up-and downregulated genes in Arabidopsis and

1007 Capsella microspores.

1008 Supplemental dataset 3. Up-and downregulated genes in Capsella microspores

1009 overlapping with regions losing 21/22-or 24-nt siRNAs in Cr nrpd1 microspores.

1010 Supplemental dataset 4. Up-and downregulated genes in Arabidopsis

1011 microspores overlapping with regions losing 21/22-or 24-nt siRNAs in At nrpd1

1012 microspores.

1013

1014 Supplemental table 1. Primer list.

1015 Supplemental table 2. Quality of sequencing samples. 
A

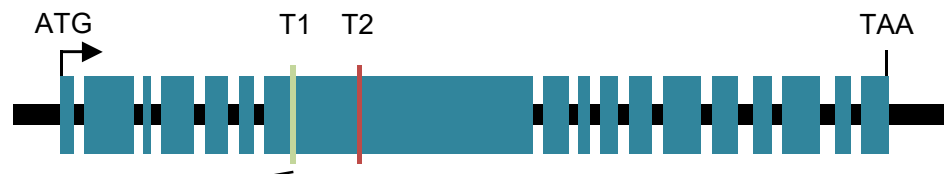

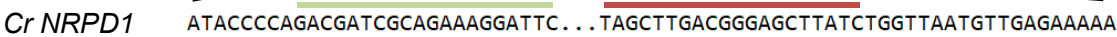

Cr nrpd1

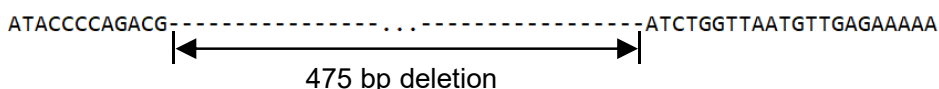

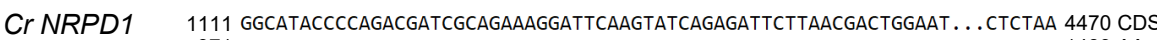

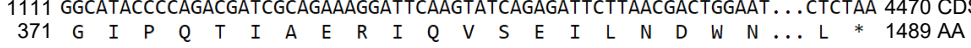

Cr nrpd1

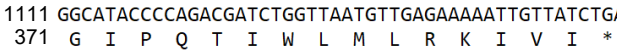
1158 CDS
385 AA

B

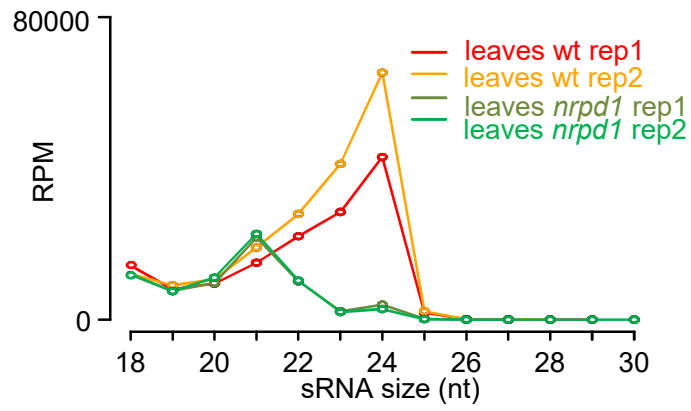

$\mathrm{C}$
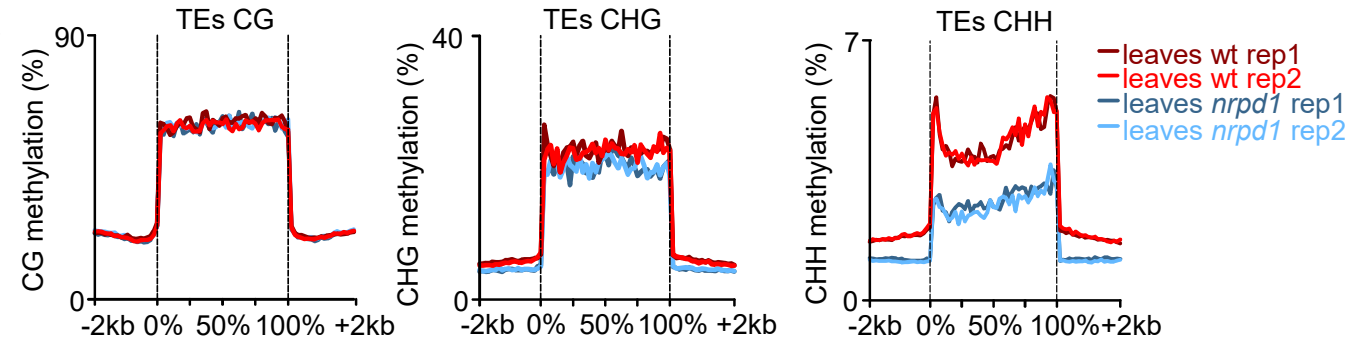

Figure 1. Disruption NRPD1 in Capsella impairs 24-nt siRNA formation and RdDM.

(A) Deleted genomic region in Capsella NRPD1 at $1634-2108$ bp (genomic sequence). Target 1

(T1) and target (T2) sequences of Crispr/Cas9 are indicated.

(B) Profile of TE-derived sRNAs in Capsella wild-type (wt) and nrpd1 leaves.

(C) DNA methylation levels at TEs in Capsella wt and nrpd1 leaves. 
A
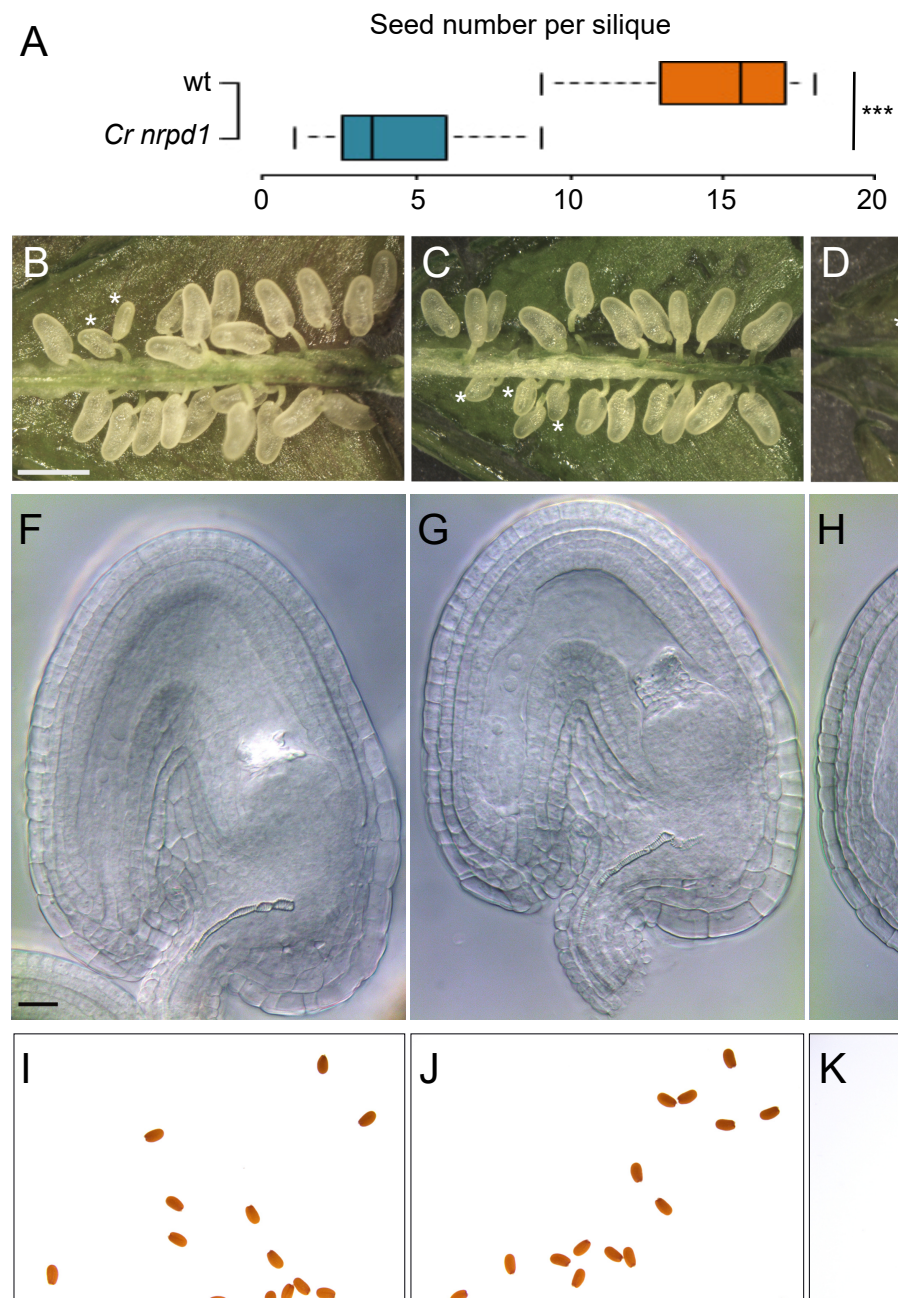

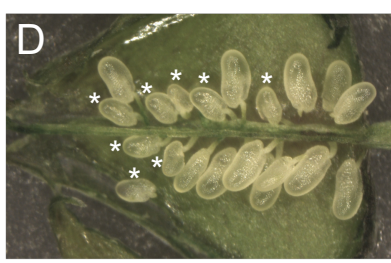

$\mathrm{E}$
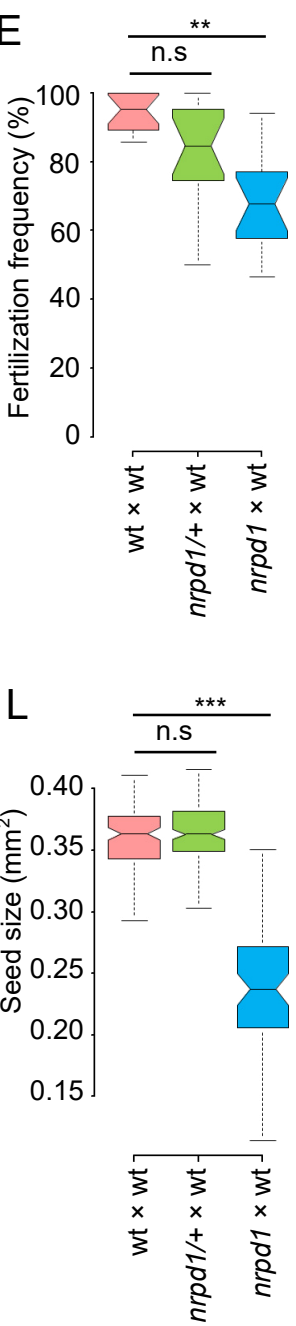

Figure 2. Loss of NRPD1 in Capsella affects female fertility and causes a reduction of seed size.

(A) Total seed numbers per silique in wt (11 siliques) and $\mathrm{Cr}$ nrpd1 mutant (24 siliques) plants. ${ }^{* * *} p$-value $<0.001$ (Student t-test). Siliques at 2 days after pollination (DAP) from (B) wt $\times w t$,

(C) $n r p d 1 /+\times$ wt, (D) nrpd1 $\times$ wt crosses. Bar (B-D): $1 \mathrm{~mm}$. Asterisks mark unfertilized ovules.

(E) Fertilization frequency in indicated crosses. 16 siliques per cross combination were analyzed. ** $p$-value $<0.01$ (Student t-test). Ovules at 2 days after emasculation of $(F) w t,(G) n r p d 1 /+$, (H) nrpd1 plants. Bar (F-H): $20 \mu \mathrm{m}$. Seeds harvested from (I) wt $\times$ wt, (J) nrpd1/+ $\times$ wt, $(\mathrm{K}) n r p d 1$ $x$ wt crosses. Bar (I-K): $1 \mathrm{~mm}$. (L) Seed size of mature seeds derived from wt $\times$ wt $(n=120)$, $n r p d 1 /+\times$ wt $(\mathrm{n}=149), n r p d 1 \times$ wt $(\mathrm{n}=64) .{ }^{* * *} \mathrm{p}$-value $<0.001$ (Student t-test). n.s, not significant. 
bioRxiv preprint doi: https://doi.org/10.1101/863522; this version posted December 3, 2019. The copyright holder for this preprint (which was not certified by peer review) is the author/funder, who has granted bioRxiv a license to display the preprint in perpetuity. It is made available

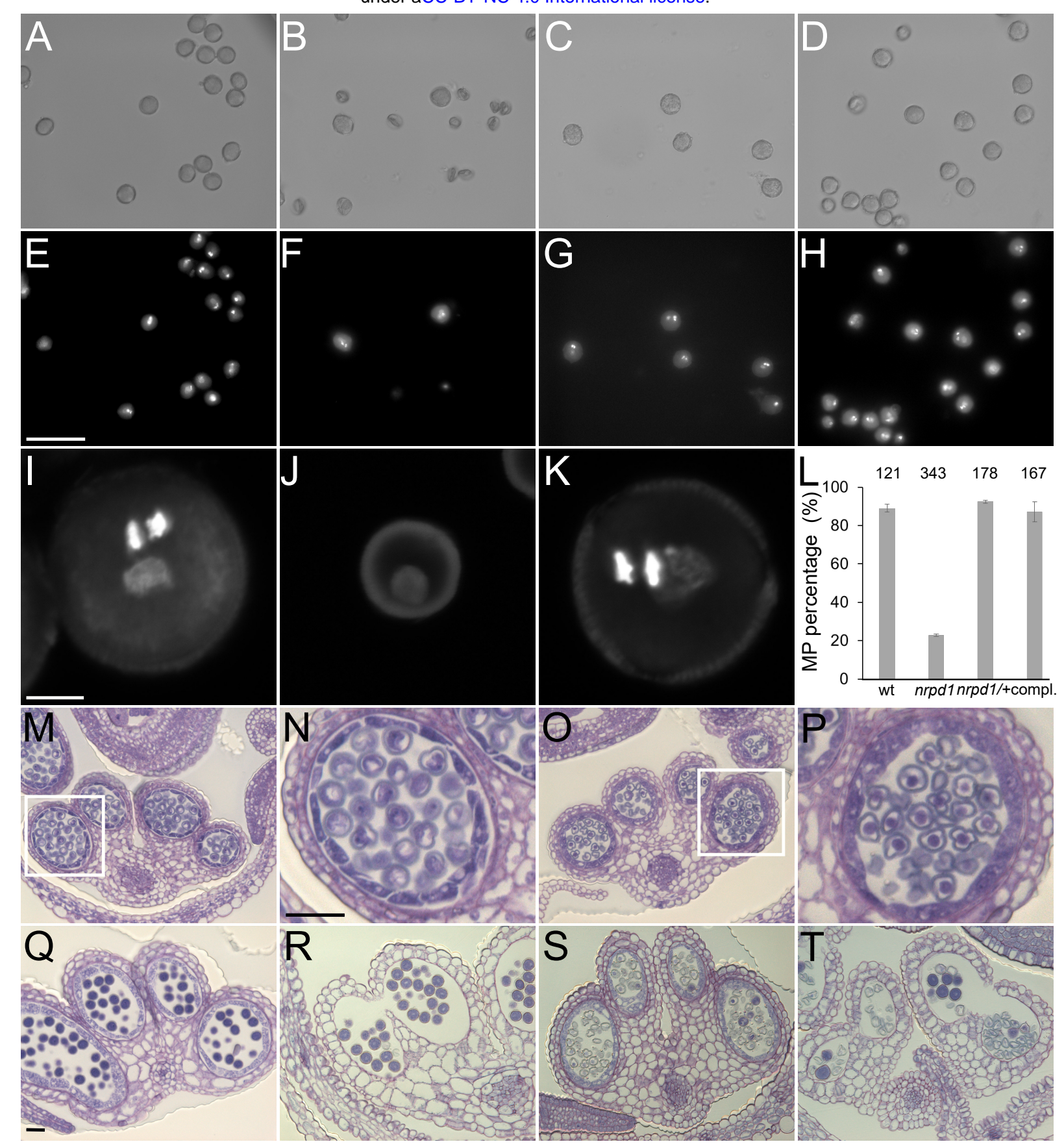

Figure 3. Cr nrpd1 pollen arrest at the microspore stage.

(A - D) Bright field and $(E-H)$ corresponding DAPI staining of manually dissected pollen from anthers at stage 12/13. Pollen of wild type (wt) ( $A$ and E), Cr nrpd1 homozygotes (B and F), Cr nrpd1 heterozygotes ( $C$ and $G$ ), and a complemented line $(D$ and $H)$. Bar $(A-H): 50 \mu \mathrm{m}$. Confocal images of DAPI stained pollen of wt (I), Cr nrpd1 homozygotes (J), and Cr nrpd1 heterozygotes (K). Bar (I-K): $5 \mu \mathrm{m}$. (L) Percentage of mature pollen (MP) in anthers dissected at stage 12/13 from wt, Cr nrpd1 homozygotes (nrpd1), Cr nrpd1 heterozygotes (nrpd1/+) and a complemented line (compl.), numbers of pollen counted in each genotype were shown on top of the bars accordingly. Microsporangia cross-sections stained with Toluidine Blue at anther stage 8 ( $\mathrm{M}$ and $\mathrm{O}$ ), 11 ( $\mathrm{Q}$ and $\mathrm{S}$ ), and 12 (R and $\mathrm{T}$ ) of wt (M, $\mathrm{Q}$ and $\mathrm{R}$ ) and $\mathrm{Cr} n \operatorname{nrpd} 1$ (O, S and $\mathrm{T}$ ). $\operatorname{Bar}(\mathrm{M}, \mathrm{O}, \mathrm{Q}-\mathrm{T}): 50 \mu \mathrm{m}$. Insets in $(\mathrm{M})$ and $(\mathrm{O})$ are shown enlarged in $(\mathrm{N})(\mathrm{P})$, respectively. Bar $(\mathrm{N}$ and $\mathrm{P})$ : $50 \mu \mathrm{m}$. wt, wild type. 
bioRxiv preprint doi: https://doj.org/10.1101/863522; this version postod December 3, 2019. The copyright holder for this preprint (which was not certified by peer revie sis

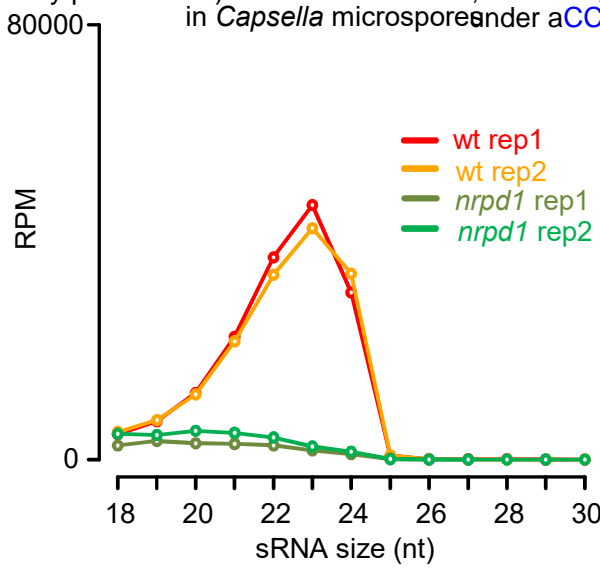

C

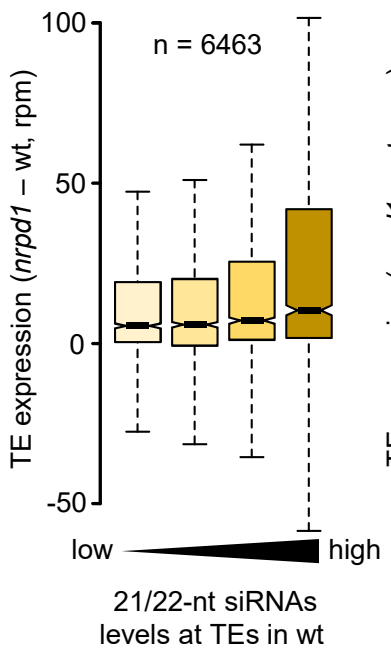

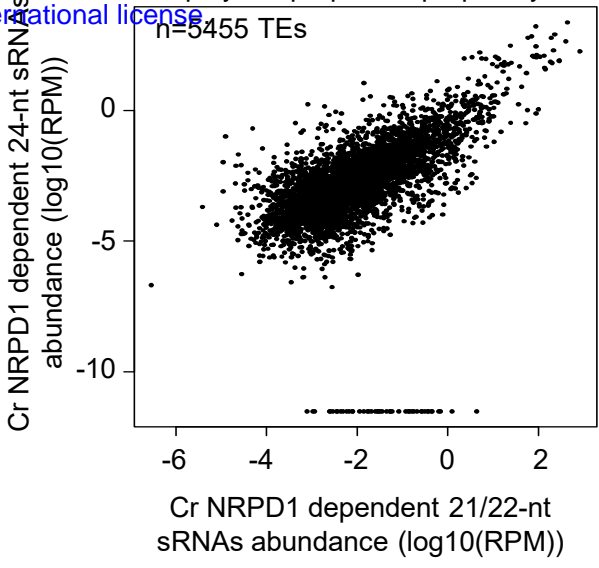

D

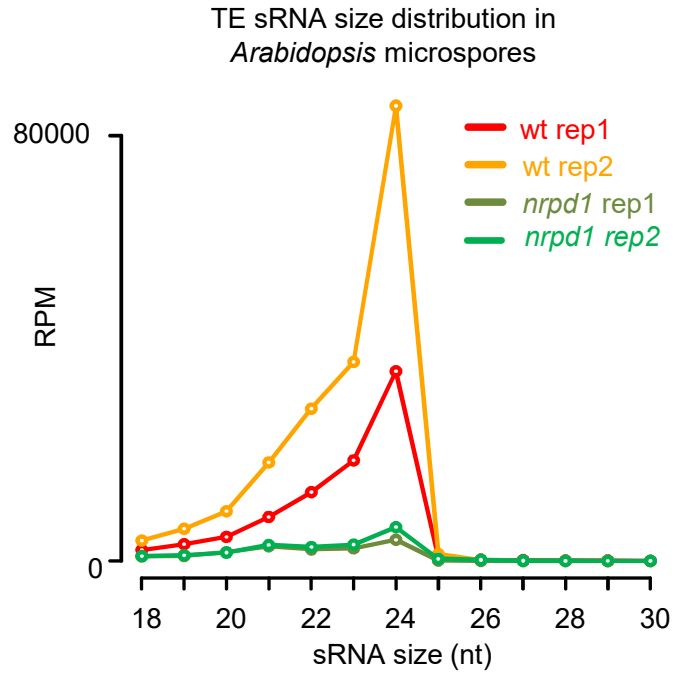

Figure 4. NRPD1 is required for 21-24-nt siRNAs in Capsella microspores.

(A) Profile of TE-derived siRNAs in Capsella wild-type (wt) and nrpd1 microspores.

(B) Abundance of TE-derived Cr NRPD1-dependent 21/22-nt siRNAs and 24-nt siRNAs in Capsella microspores. Values are indicated as the $\log 10$ of the average RPM of both libraries. Each dot represents one TE for a total of 5455 TEs. The correlation has been tested by a Spearman test (correlation coefficient 0.6872 ).

(C) Loss of 21/22-nt and 24-nt siRNAs at TEs associates with increased transcript level of TEs in Cr nrpd1 microspores. Increasing accumulation of siRNAs over TEs is plotted from low to high levels of accumulation. Only TEs with siRNAs more in wt than in $\mathrm{Cr} n r p d 1$ are represented. Differences between first and last categories are significant $(p=3.4 \mathrm{e}-13$ and $1.4 \mathrm{e}-9$, respectively, Wilcoxon test).

(D) sRNA profile of TE-derived sRNA from Arabidopsis wt and nrpd1 microspores. 
bioRxiv preprint doi: https://doi.org/10.1101/863522; this version posted December 3, 2019. The copyright holder for this preprint (which was not certified by peer review) is the author/funder, who has granted bioRxiv a license to display the preprint in perpetuity. It is made available

A

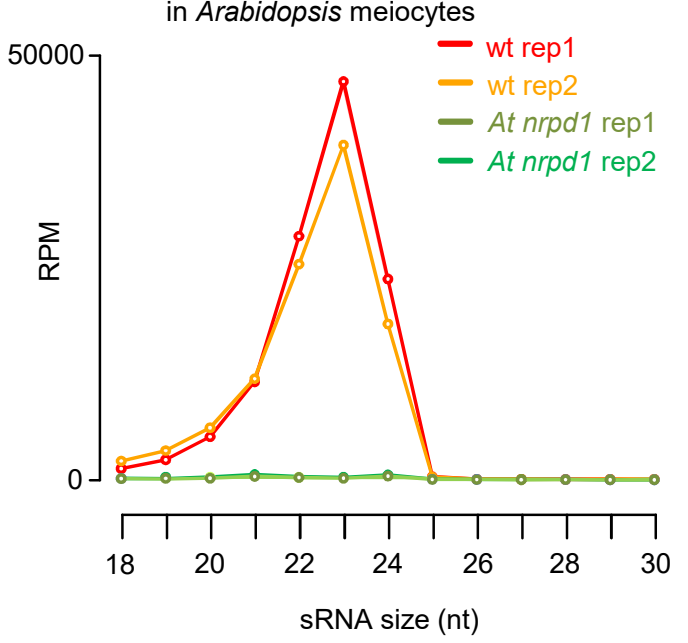

C

TEs producing sRNAs in microspores and meiocytes

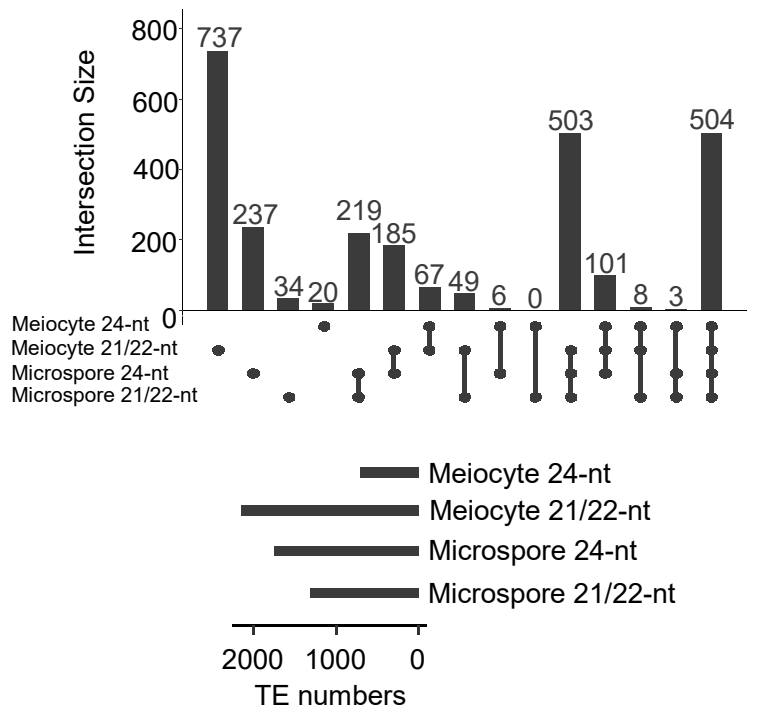

B

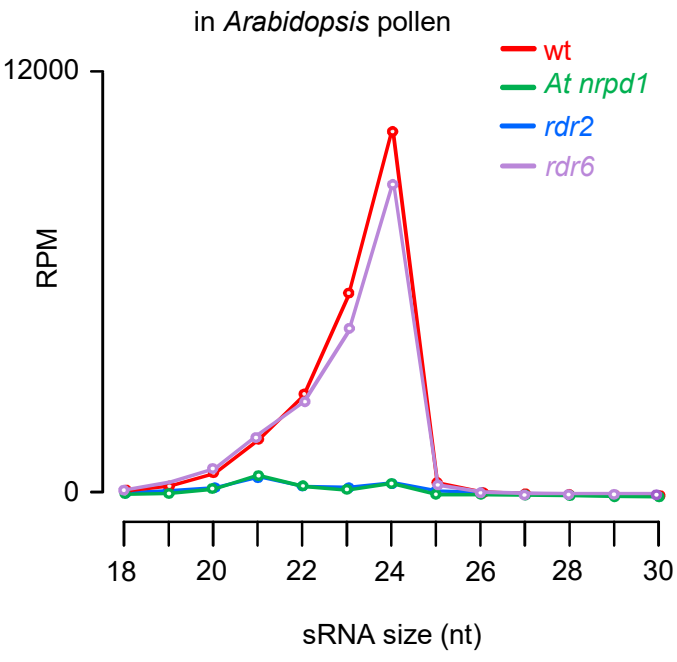

TEs producing sRNAs in microspores and mature pollen grains

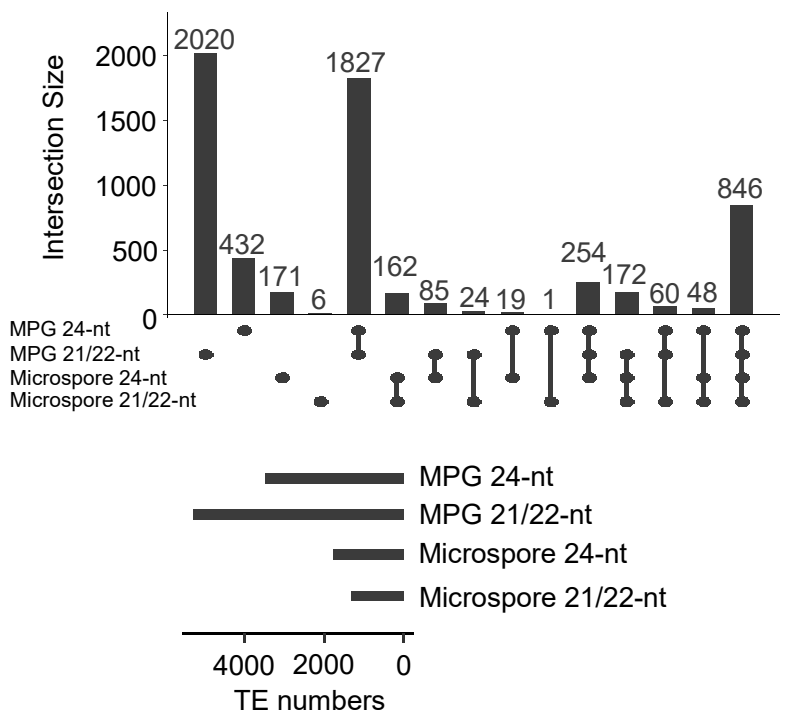

Figure 5. Meiocytes, microspores and mature pollen grain accumulate overlapping sets of SiRNAs.

(A) TE-derived siRNA distribution in Arabidopsis meiocytes of the indicated genetic background (data from Huang et al., 2019).

(B) TE-derived siRNA distribution in Arabidopsis pollen grains of the indicated genetic background.

(C) Upset plot showing the overlap of TEs accumulating 21/22-nt siRNAs or 24-nt siRNAs in

Arabidopsis microspores and meiocytes (data from Huang et al., 2019).

(D) Upset plot showing the overlap of TEs accumulating 21/22-nt siRNAs or 24-nt siRNAs in

Arabidopsis microspores and mature pollen grain (MPG) (data from Martinez et al., 2018). 
${ }^{30} \mathrm{n}=3927 \quad{ }^{T} \quad 30, \mathrm{i}=4259$

bioRxiv preprint doi: https://dọi.org/10.11 1//863522; this version post

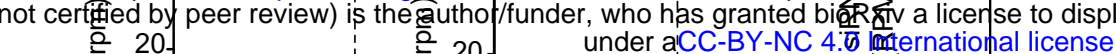
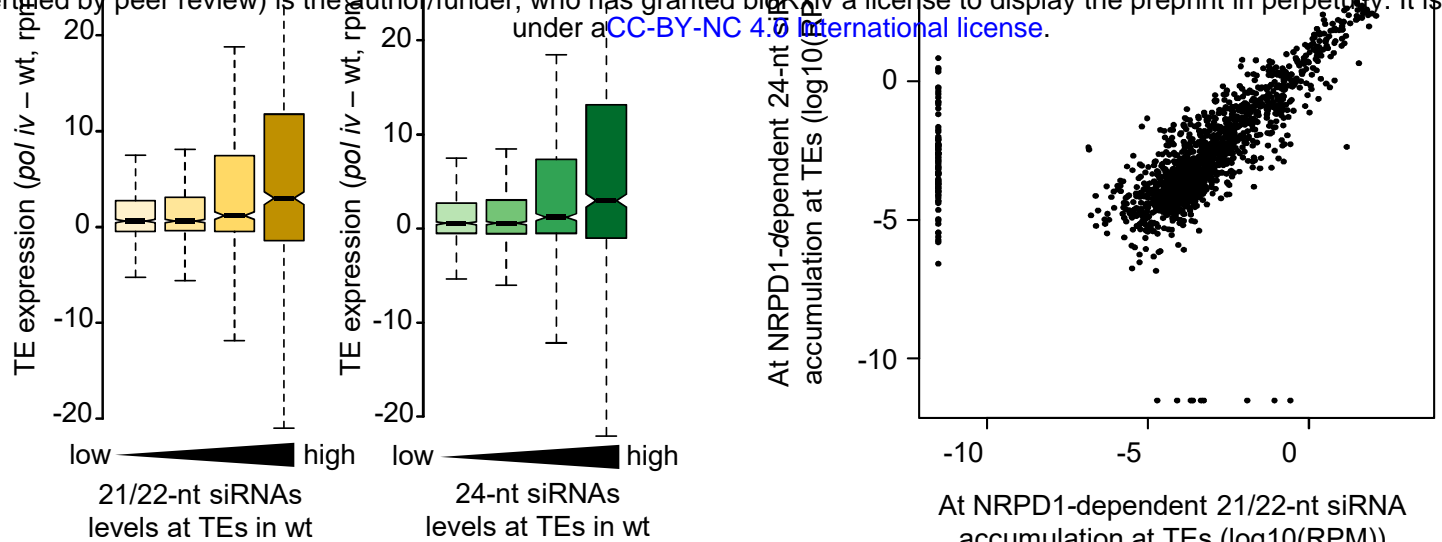

At NRPD1-dependent 21/22-nt siRNA accumulation at TEs $(\log 10(\mathrm{RPM}))$

C

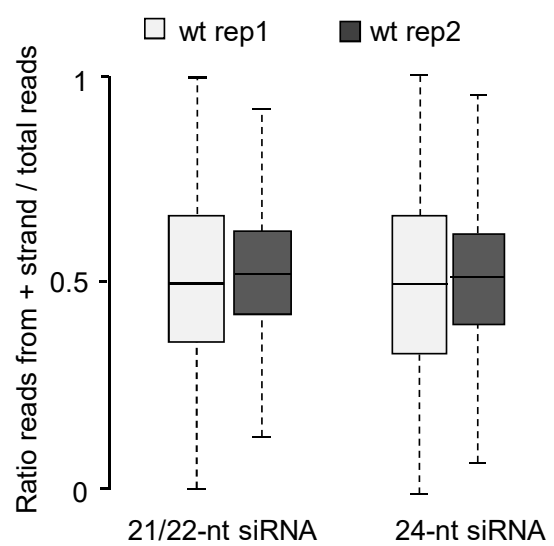

$\mathrm{D}$

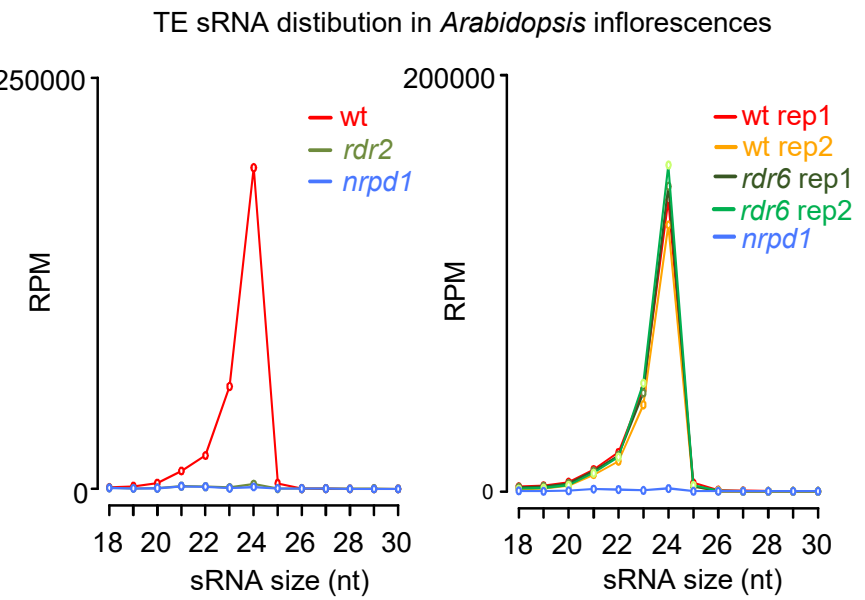

$E$

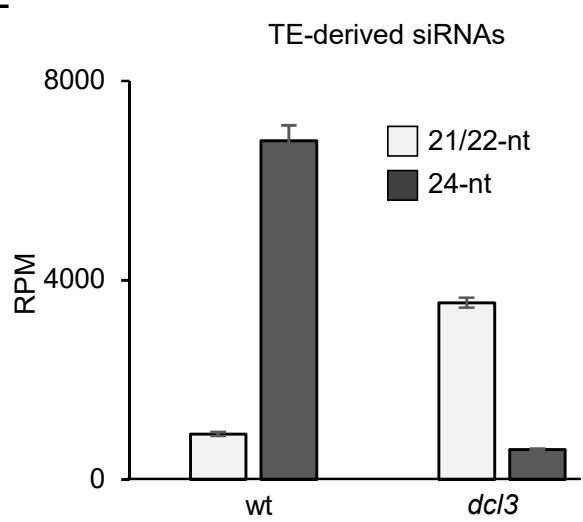

$\mathrm{F}$

miRNAs

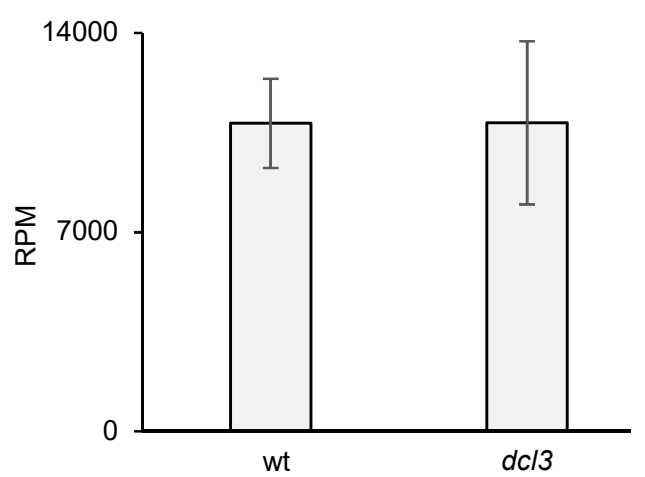

Figure 6. Pol IV/RDR2 generate templates for 21-24-nt siRNAs.

(A) Loss of At NRPD1 dependent 21/22-nt easiRNAs associates with increased transcript levels of TEs in Arabidopsis microspores. Increasing accumulation of siRNAs over TEs is plotted from low to high levels of accumulation. In both plots, siRNAs levels at TEs in wt increase from left to right in quantiles. Differences between first and last categories are significant $(p=2.6 \mathrm{e}-10$ and $1.5 \mathrm{e}-14$, respectively, Wilcoxon test).

(B) Abundance of At NRPD1-dependent 21/22-nt siRNAs and 24-nt siRNAs at TEs in Arabidopsis microspores. Values are indicated as $\log 10$ of the average reads per million (RPM) of both libraries. Each dot represents one TE for a total of 1504 TEs. The correlation has been tested by a Spearman test (correlation coefficient 0.7686 ).

(C) Plots showing the distribution of the ratio of the number of reads mapped against the positive strand to the total number of mapped reads. Left plots shows analysis for 21/22-nt reads, right plot for 24-nt reads.

(D) TE-derived siRNA distribution in inflorescences of $r d r 2$ (left panel; data from Zhai et al., 2015) and $r d r 6$ (right panel; data from Panda et al., 2016).

(E) Average total 21/22-nt or 24-nt reads mapping against TEs in wt or dc/3 libraries (data from Li et al., 2015). Reads were normalized to show RPM values.

(F) Average total 21/22-nt reads mapping against miRNAs in wt or $d c / 3$ libraries (data from Li et al., 2015). Reads were normalized to show RPM values. 
A

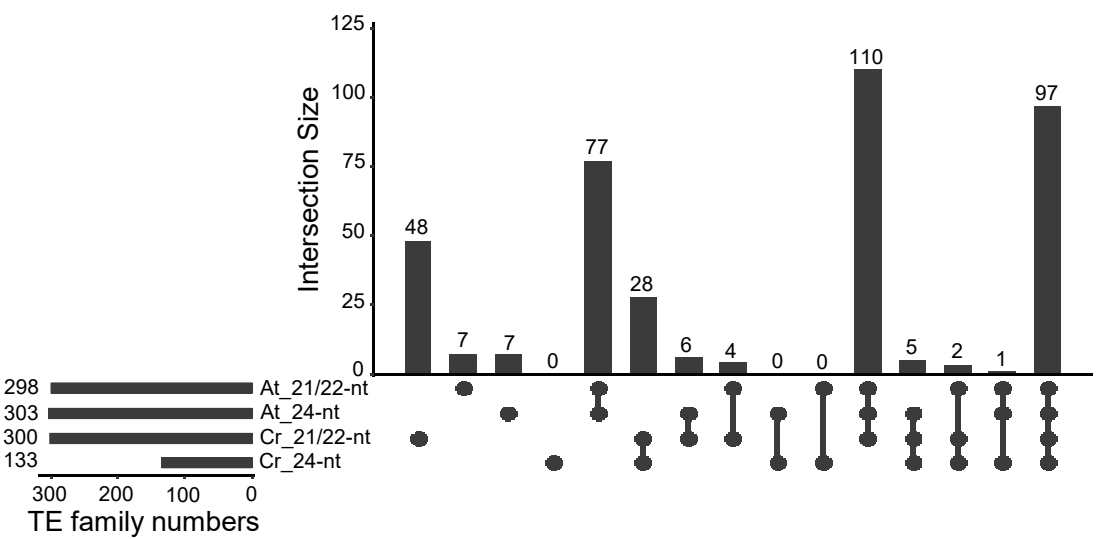

B

21/22-nt Pol IV TE-siRNAs

C

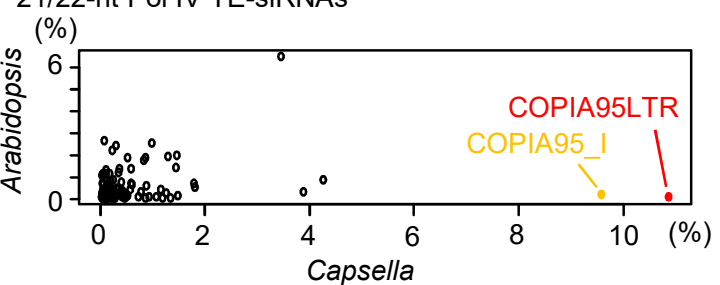

24-nt Pol IV TE-siRNAs
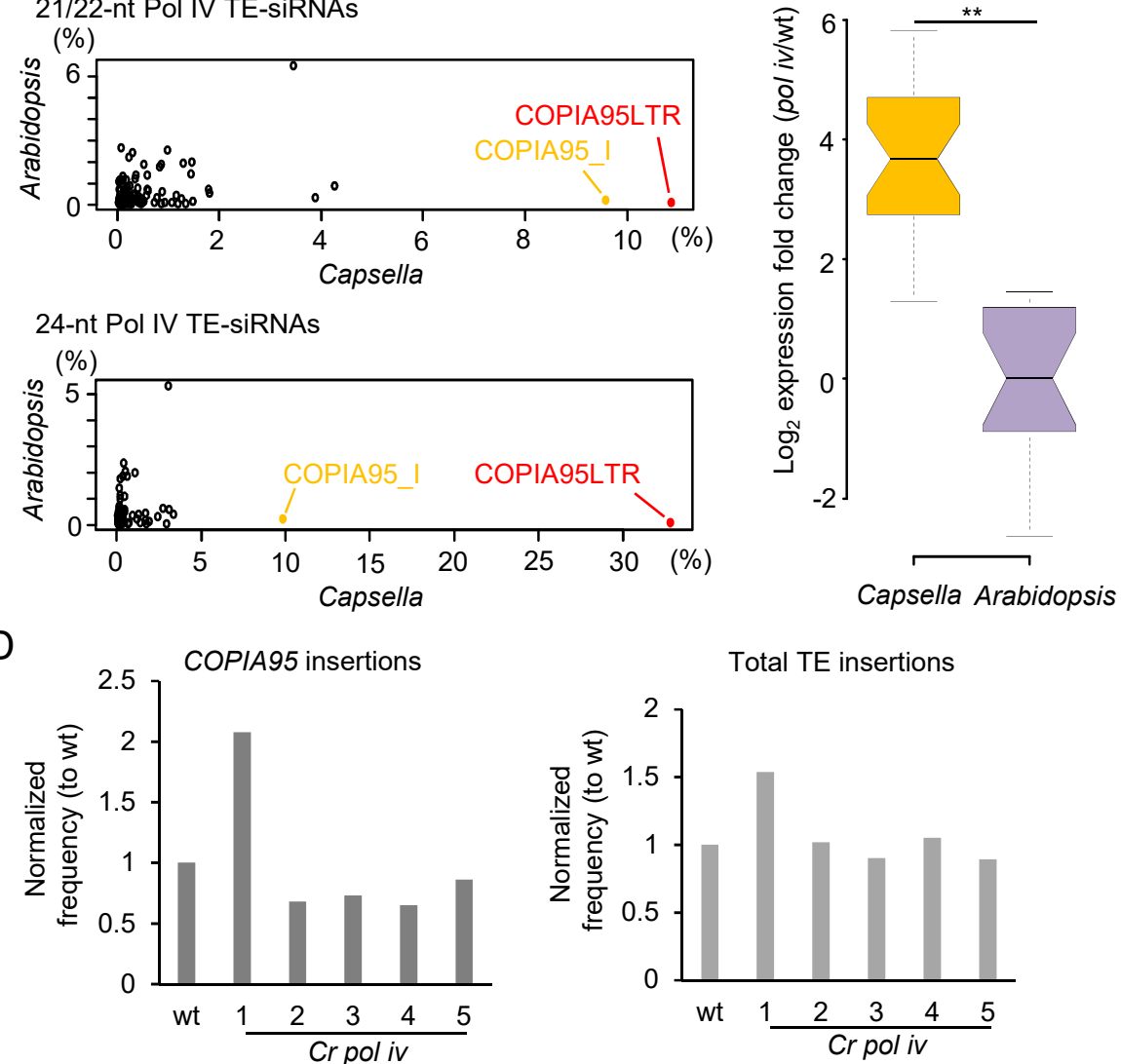

Figure 7. COPIA95-siRNAs are highly enriched in Capsella microspores.

(A) Upset plots of TE families accumulating Pol IV-dependent 21/22-nt and 24-nt siRNAs in Arabidopsis (At) and Capsella $(\mathrm{Cr})$.

(B) Proportions of Pol IV-dependent 21/22-nt and 24-nt siRNAs accumulating at specific TE consensus sequences in relation to all TE-siRNAs. Reads mapping to COPIA95 longterminal repeats (LTR) and internal (I) sequences are highlighted in red and yellow, respectively.

(C) Log2 expression fold change of mRNAs for COPIA95 elements in nrpd1 mutant microspores of Arabidopsis and Capsella compared to the corresponding wild type. ${ }^{* *} \mathrm{p}<0.01$ (Student's t-test).

(D) Relative number of COPIA95 insertions (left panel) and total TE insertions (right panel) compared to the corresponding wild-type control in five progenies of homozygous Cr nrpd1. 
A Genes losing 21/22-nt siRNAs

in Capsella nrpd1 microspores Upregulated genes (3194) (3471)

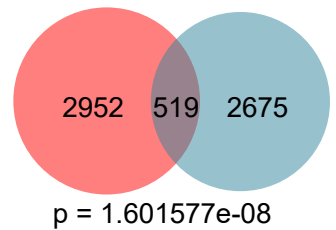

Genes losing 24-nt siRNAs in

Capsella nrpd1 microspores Upregulated genes (3194) (3346)

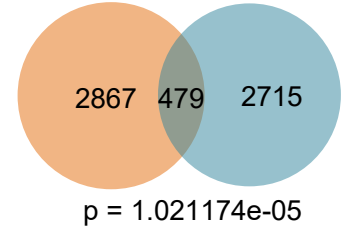

B
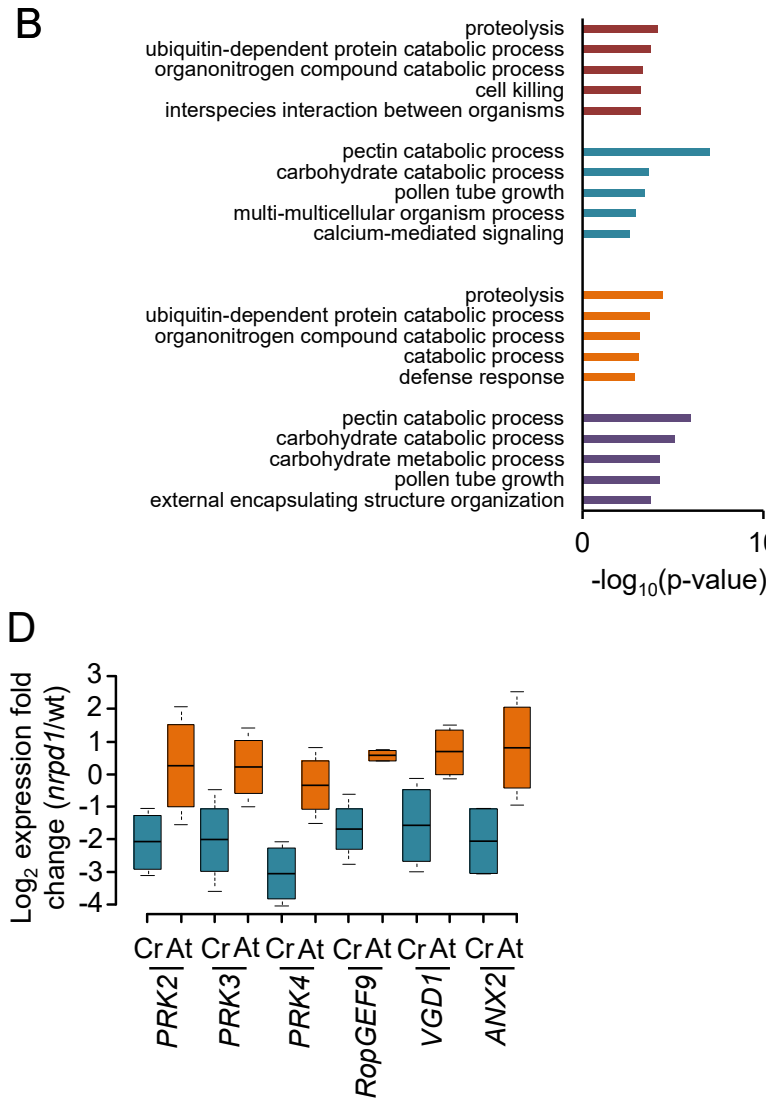

Genes losing 21/22-nt siRNAs

in Capsella nrpd1 microspores Downregulated genes (1985)

(3471)

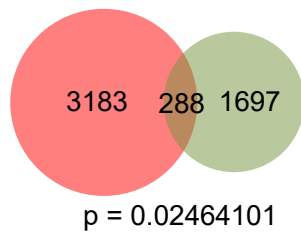

Genes losing 24-nt siRNAs in

Capsella nrpd1 microspores Downregulated genes (1985)

(3346)

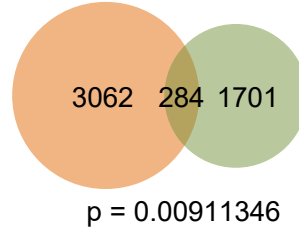

Upregulated genes losing 21/22-nt siRNAs

Downregulated genes losing 21/22-nt siRNAs

Upregulated genes losing 24-nt siRNAs

Downregulated genes losing 24-nt siRNAs
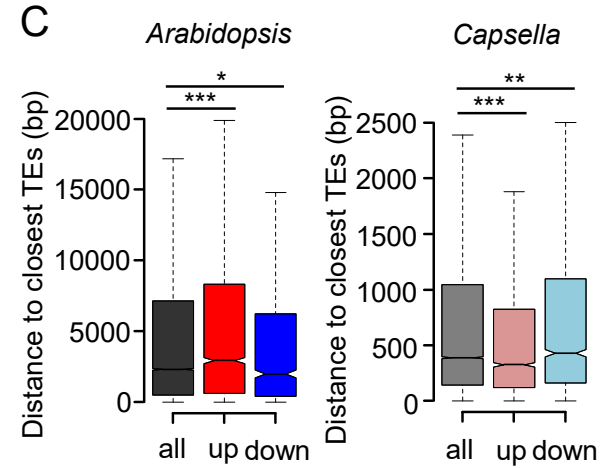

$E$

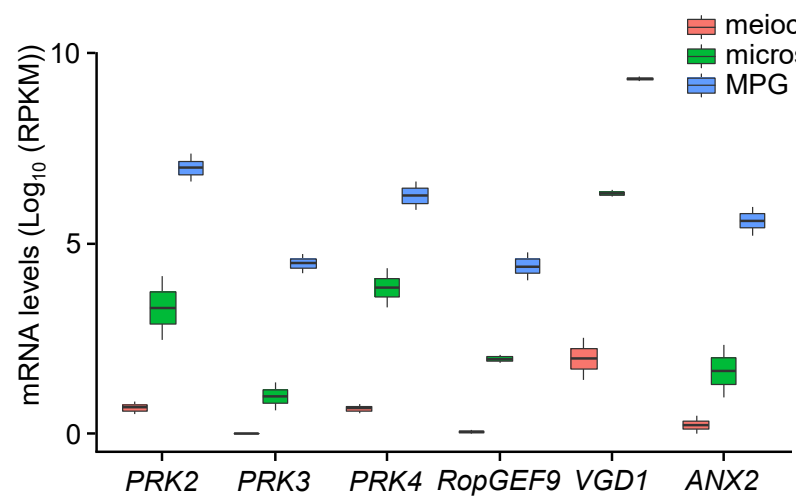

Figure 8. Deregulated genes differ in Arabidopsis and Capsella nrpd1 mutant microspores.

(A) Venn diagrams showing overlap of deregulated genes $\left(\| \log _{2}\right.$ fold change $\left.\mid>1, p<0.05\right)$ in nrpd1 microspores of Capsella and genes losing 21/22-nt and 24-nt siRNAs at 2kb up-and downstream and gene body $\left(\log _{2}\right.$ fold change $\left.<-1, p<0.05\right)$ in Capsella $n r p d 1$ microspores. (B) Enriched gene ontologies (GO) for biological processes of intersected genes losing siRNAs and deregulated genes in Capsella nrpd1 microspores. Top 5 GOs of each analysis are shown. (C) Distance of Arabidopsis and Capsella genes to closest TEs. All: all genes, up: significantly upregulated genes, down: significantly downregulated genes. ${ }^{*} p<0.05$, ${ }^{* *} p<0.01,{ }^{* * *} p<0.001$, n.s, not significant. (Statistical analysis: Wilcoxon test).

(D) $\log _{2}$ expression fold change of, PRK2, PRK3, PRK4, RopGEF9, VGD1 and ANX2 genes in nrpd1 microspores compared to wild type (wt) in Capsella $(\mathrm{Cr})$ and Arabidopsis (At).

(E) mRNA levels of PRK2, PRK3, PRK4, RopGEF9, VGD1 and ANX2 in Arabidopsis wild-type meiocytes, microspores and mature pollen grain (MPG). We added plus1 to all values to avoid negative $\log _{10}$ values. 


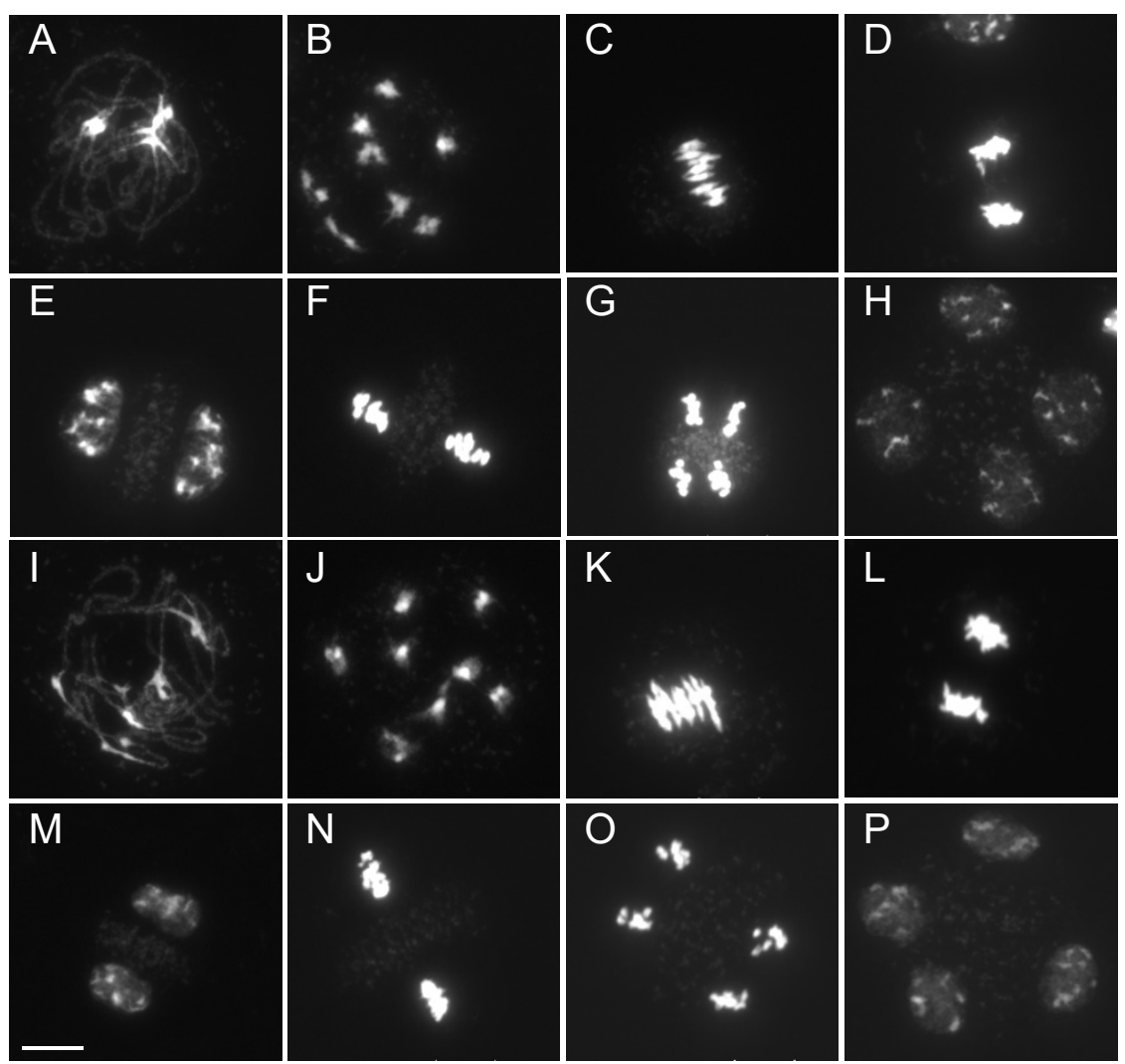

Supplemental Figure 1. Meiosis is not affected in Capsella nrpd1. Supports Figure 2.

Meiosis in Capsella wild-type (A - H) and $n r p d 1$ (I - P) plants. A and I, pachytene. B and $\mathrm{J}$, diakinesis. $\mathrm{C}$ and $\mathrm{K}$, metaphase I. D and L, telophase I. E and $\mathrm{M}$, prophase II. $\mathrm{F}$ and $\mathrm{N}$, metaphase II. $\mathrm{G}$ and $\mathrm{O}$, anaphase II. $\mathrm{H}$ and $\mathrm{P}$, telophase II. Bar: $5 \mu \mathrm{m}$. 
bioRxiv preprint doi: https://doi.org/10.1101/863522; this version posted December 3, 2019. The copyright holder for this preprint (which was not certified by peer review) is the author/funder, who has granted bioRxiv a license to display the preprint in perpetuity. It is made available under aCC-BY-NC 4.0 International license.
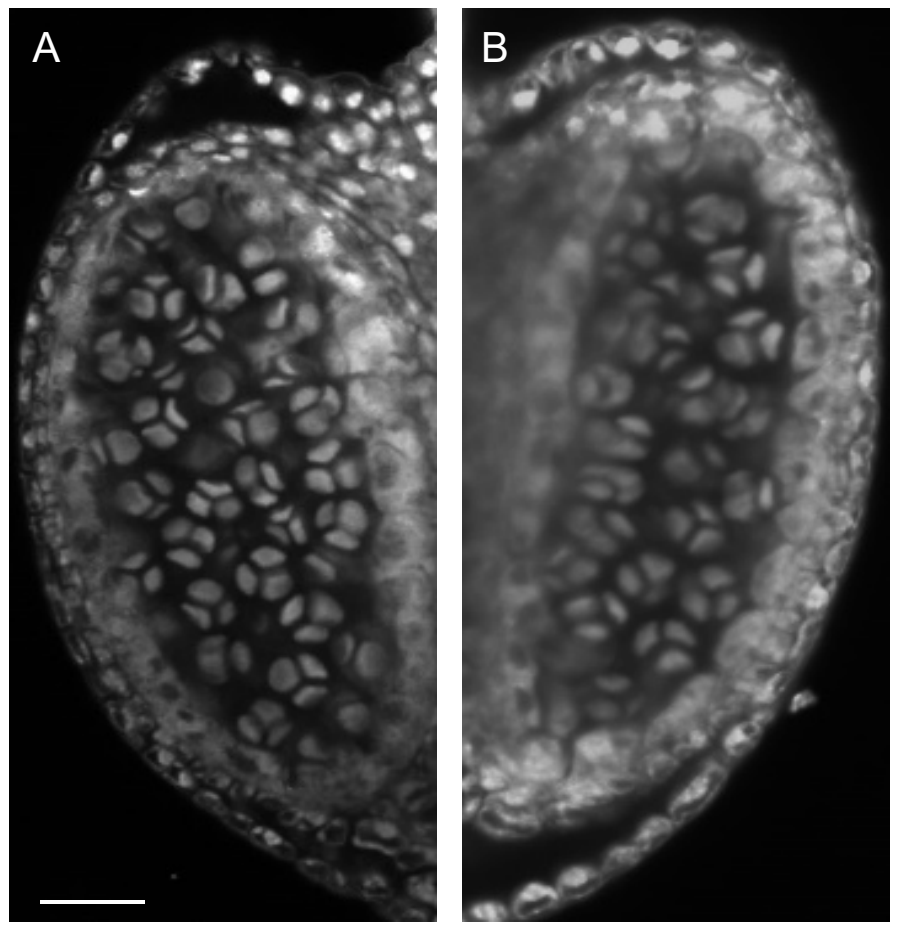

Supplemental Figure 2. Normal tetrad formation in Capsella wild type (A) and $n r p d 1$ (B). Supports Figure 2.

Shown are whole mount confocal images. Bar: $20 \mu \mathrm{m}$. 
A

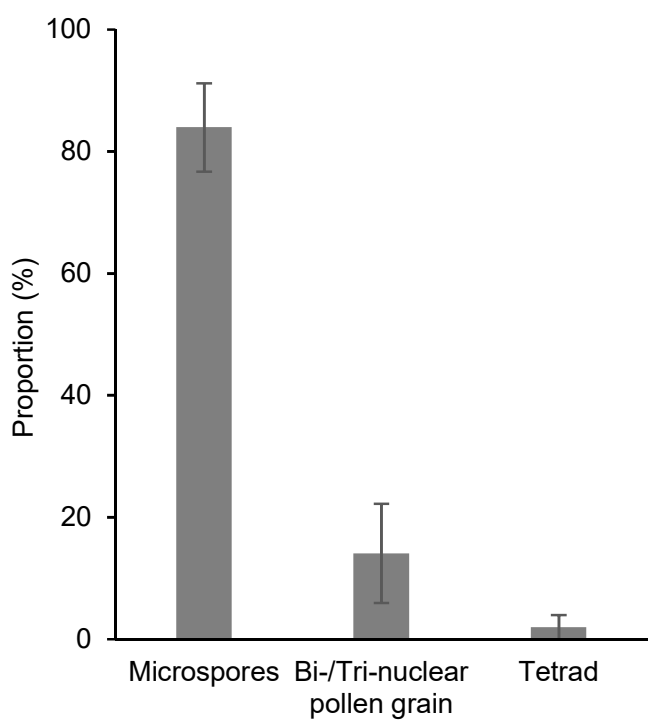

B

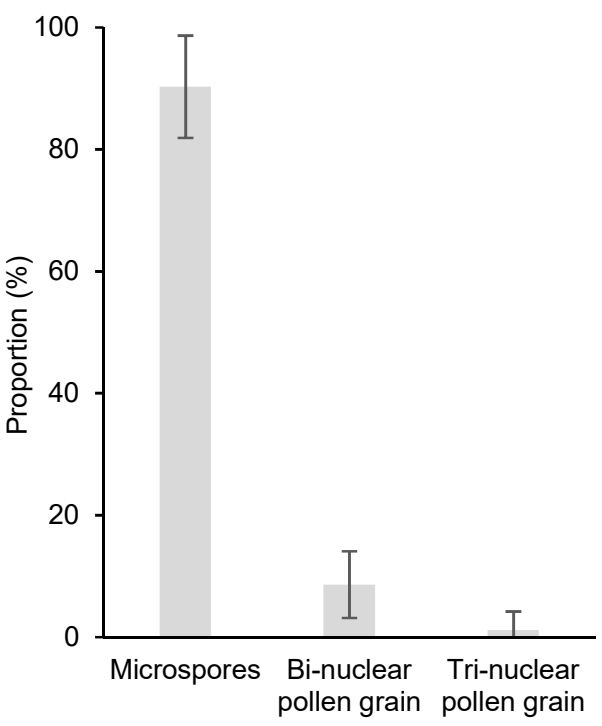

Supplemental Figure 3. Average purity of Capsella and Arabidopsis microspore extractions. Supports Figure 4.

Microspore extractions of Capsella (A) and Arabidopsis (B) were tested by DAPI staining and the $\mathrm{B} 2$ and $\mathrm{B} 1$ fractions were selected as the fractions containing the highest proportion of microspores in Capsella and Arabidopsis, respectively. Shown is the average percentage of four and eight independent extractions in Capsella and Arabidopsis, respectively. Error bars show standard deviation. 
bioRxiv preprint doi: https://doi.org/10.1101/863522; this version posted December 3, 2019. The copyright holder for this preprint (which was not certified by peer review) is the author/funder, who has granted bioRxiv a license to display the preprint in perpetuity. It is made available under aCC-BY-NC 4.0 International license.

A

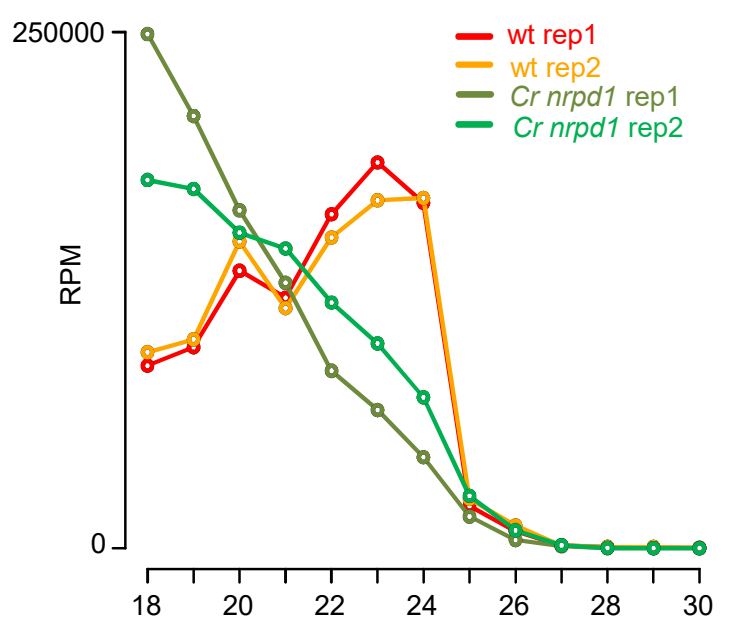

B

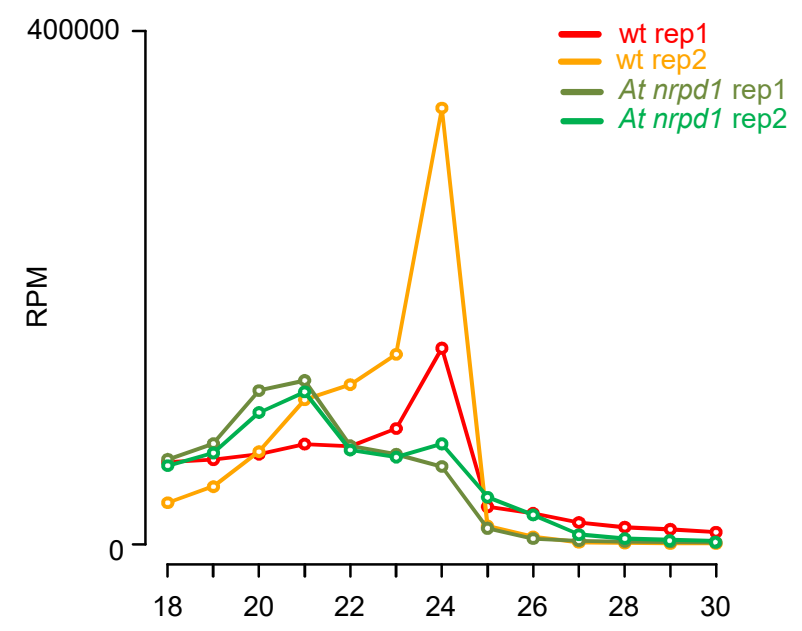

Supplemental Figure 4. Profile of total sRNAs in Capsella (A) and Arabidopsis (B) microspores. Supports Figure 4. 
bioRxiv preprint doi: https://doi.org/10.1101/863522; this version posted December 3, 2019. The copyright holder for this preprint (which was not certified by peer review) is the author/funder, who has granted bioRxiv a license to display the preprint in perpetuity. It is made available under aCC-BY-NC 4.0 International license.
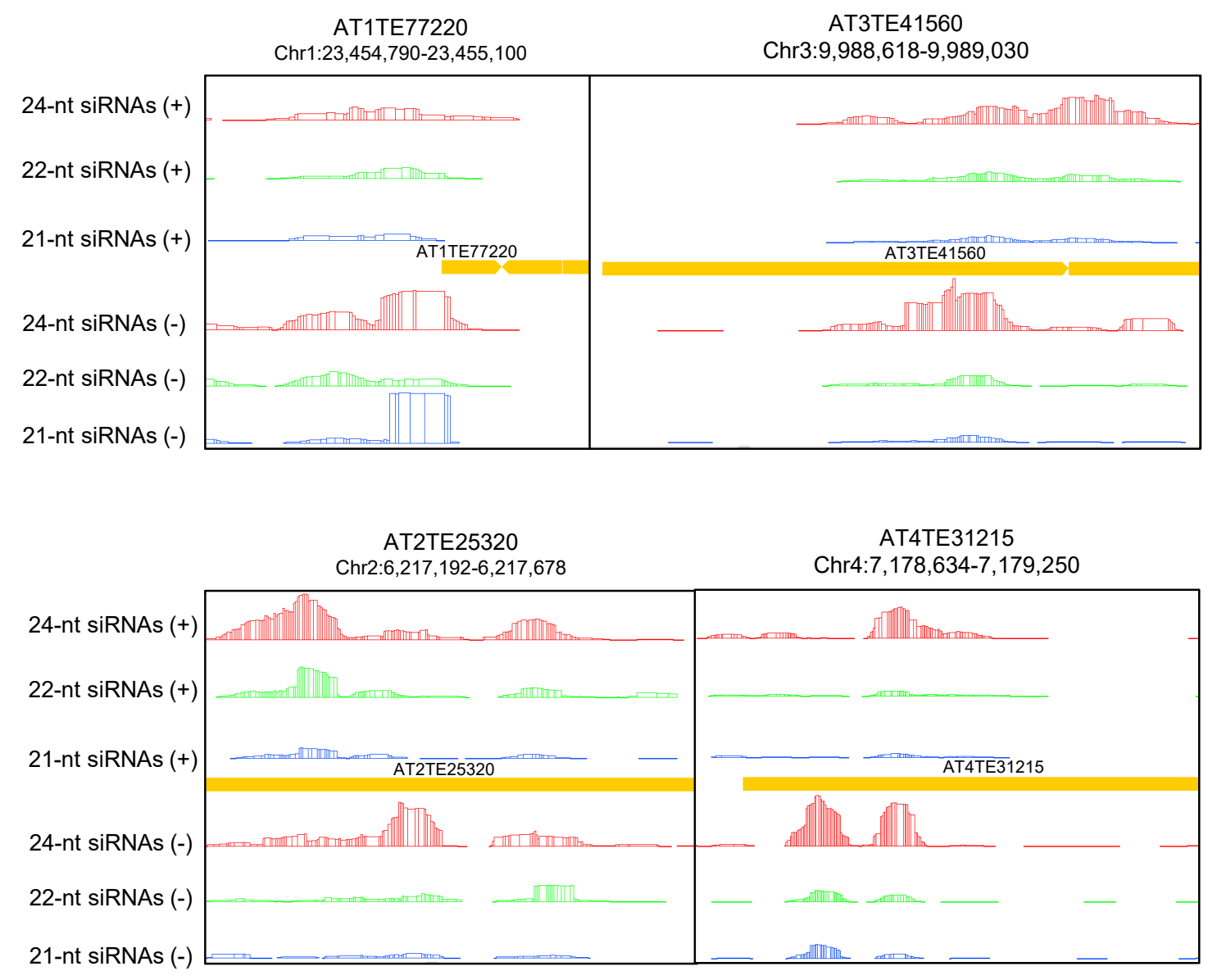

Supplemental Figure 5. Examples of four loci producing Pol IV-dependent siRNAs in Arabidopsis. Supports Figure 6.

Bars represent normalized reads. The color indicates the length of the analyzed reads: red 24-nt, blue 22-nt, and green 21-nt. The DNA strand is indicated by the (+) or (-). TE sequences are represented in yellow. 

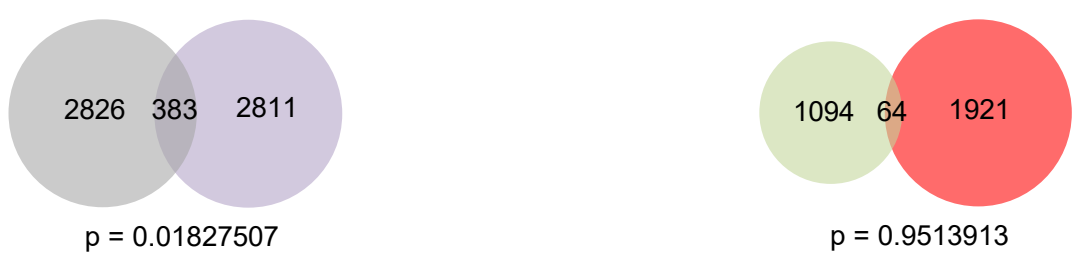

C

$$
p=0.01827507
$$

$$
p=0.9513913
$$

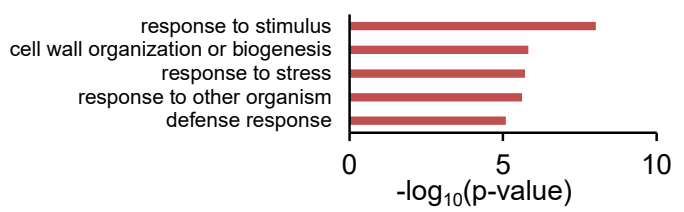

Upregulated genes shared in Arabidopsis and Capsella
D Genes losing 21/22-nt siRNAs in Arabidopsis nrpd1 microspores Upregulated genes (3209) (3123)

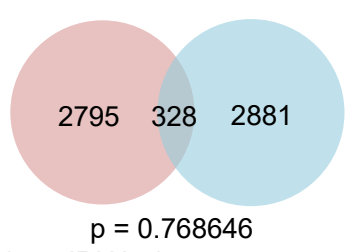

Genes losing 24-nt siRNAs in Arabidopsis nrpd1 microspores Upregulated genes (3209) (3302)

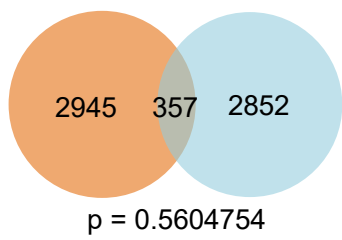

Genes losing 21/22-nt siRNAs in

Arabidopsis nrpd1 microspores Downregulated genes (1158) (3123)

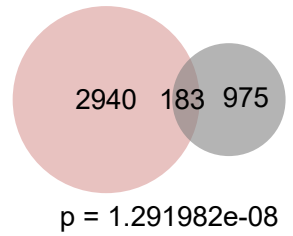

Genes losing 24-nt siRNAs in

Arabidopsis nrpd1 microspores Downregulated genes (1158) (3302)

E

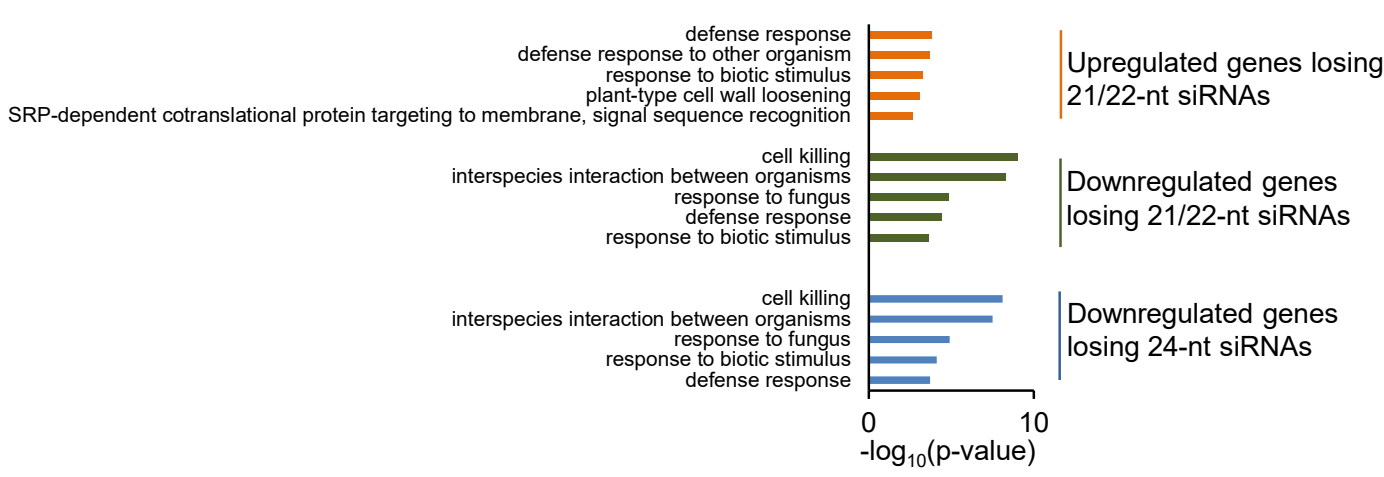

\section{Supplemental Figure 6. Deregulated genes in Arabidopsis nrpd1 mutant microspores.}

\section{Supports Figure 8.}

(A) Venn diagram showing overlap of upregulated genes in nrpd1 microspores of Capsella and Arabidopsis. Significance was determined by a hypergeometric test.

(B) Venn diagram showing overlap of downregulated genes in nrpd1 microspores of Capsella and Arabidopsis. Significance was determined by a hypergeometric test.

(C) Enriched gene ontologies (GO) for biological processes of upregulated genes shared in Arabidopsis and Capsella nrpd1 microspores. Top 5 GOs with $\mathrm{p}<0.01$ are shown.

(D) Venn diagrams showing overlap of deregulated genes $\left(\mid \log _{2}\right.$ fold change $\left.\mid>1, p<0.05\right)$ in nrpd1 microspores of Arabidopsis and genes losing 21/22-nt and 24-nt siRNAs at 2kb up-and downstream and gene body ( $\log _{2}$ fold change $<-1, p<0.05$ ) in Arabidopsis nrpd1 microspores. Significance was determined by a hypergeometric test.

(E) Enriched gene ontologies (GO) for biological processes of intersected genes losing siRNAs and deregulated genes in Arabidopsis nrpd1 microspores. Top 5 GOs with $p<0.01$ are shown. 
bioRxiv preprint doi: https://doi.org/10.1101/863522; this version posted December 3, 2019. The copyright holder for this preprint (which was not certified by peer review) is the author/funder, who has granted bioRxiv a license to display the preprint in perpetuity. It is made available under aCC-BY-NC 4.0 International license.

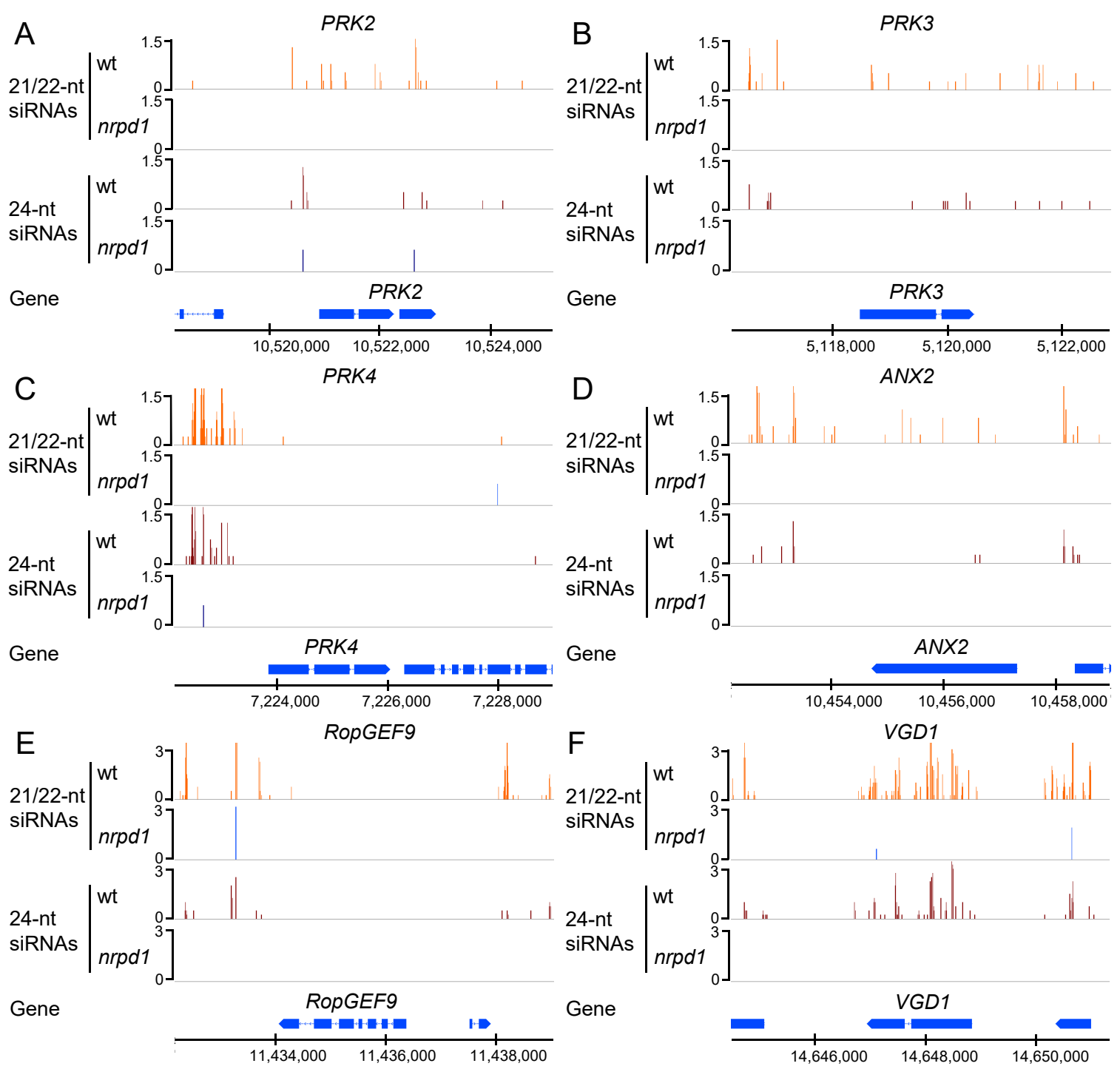

Supplemental Figure 7. Representative pollen developmental genes accumulating 21/22-nt and 24-nt siRNAs in Capsella microspores. 
bioRxiv preprint doi: https://doi.org/10.1101/863522; this version posted December 3, 2019. The copyright holder for this preprint (which was not certified by peer review) is the author/funder, who has granted bioRxiv a license to display the preprint in perpetuity. It is made available

Supplemental table 1. Primer4nSer aCC-BY-NC 4.0 International license.

\begin{tabular}{|l|l|l|}
\hline Primer names & Sequences & Application \\
\hline CrT1 NRPD1 & $\begin{array}{l}\text { ATATATGGTCTCGATTGAATCCTTTCTGCGATCGTCGTTTT } \\
\text { AGAGCTAGAAATAGC }\end{array}$ & $\begin{array}{l}\text { Generate } \\
\text { Crispr/cas9 } \\
\text { construct for } \\
\text { pol iv }\end{array}$ \\
\hline CrT2 NRPD1 & $\begin{array}{l}\text { ATTATTGGTCTCGAAACGATAAGCTCCCGTCAAGCTACAA } \\
\text { TCTCTTAGTCGACTCTAC } \\
\text { Cenerate } \\
\text { Crispr/cas9 } \\
\text { construct for } \\
\text { pol iv }\end{array}$ \\
\hline pHEE401E-Seq-F & GTTGTAAAACGACGGCCAGT & $\begin{array}{l}\text { Sequencing } \\
\text { Crispr/cas9 } \\
\text { construct }\end{array}$ \\
\hline pHEE401E-Seq-R & CAAACGCAAATGCTTTTATTCAC & $\begin{array}{l}\text { Sequencing } \\
\text { Crispr/cas9 } \\
\text { construct }\end{array}$ \\
\hline Cr-NRPD1-seq-F & GTTCTCGTGTGGTCGAATGC & $\begin{array}{l}\text { PCR for } \\
\text { identifying } \\
\text { pol iv } \\
\text { mutation }\end{array}$ \\
\hline Cr-NRPD1-seq-R & CTCATAGCAGAACCGAGCCA & $\begin{array}{l}\text { PCR for } \\
\text { identifying } \\
\text { pol iv } \\
\text { mutation }\end{array}$ \\
\hline At_NRPD1_F & GGGGACAAGTTTGTACAAAAAAGCAGGCTATGGAAGACGATTGT & $\begin{array}{l}\text { For cloning } \\
\text { Arabidopsis } \\
\text { NRPD1 }\end{array}$ \\
\hline At_NRPD1_F & GAGGAGC & $\begin{array}{l}\text { For cloning } \\
\text { Arabidopsis } \\
\text { NRPD1 }\end{array}$ \\
\hline
\end{tabular}


bioRxiv preprint doi: https://doi.org/10.1101/863522; this version posted December 3, 2019. The copyright holder for this preprint (which was not certified by peer review) is the author/funder, who has granted bioRxiv a license to display the preprint in perpetuity. It is made available

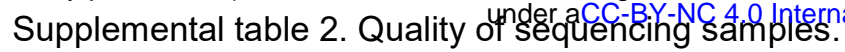

Table shows details of the sequenced samples generated in this study. Replicates are biological replicates.
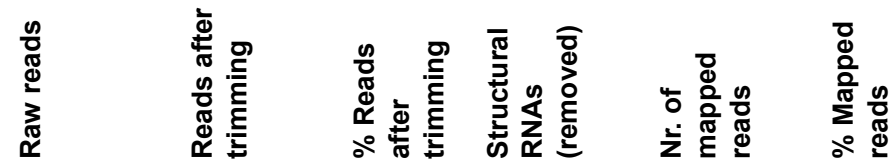

\begin{tabular}{|c|c|c|c|c|c|c|}
\hline \multicolumn{7}{|c|}{ Arabidopsis microspores } \\
\hline Col-0 Replicate 1 & 11209095 & 6753162 & 60.2 & 2489434 & 1444728 & 33.9 \\
\hline Col-0 Replicate 2 & 10665337 & 8098812 & 75.9 & 2921468 & 2771963 & 53.5 \\
\hline nrpd1 Replicate 1 & 9494296 & 6450194 & 67.9 & 3453871 & 815700 & 27.2 \\
\hline nrpd1 Replicate 2 & 12788162 & 9326033 & 72.9 & 4726264 & 1100282 & 23.9 \\
\hline \multicolumn{7}{|l|}{ Capsella microspores } \\
\hline wt Replicate 1 & 37486299 & 17981812 & 48.0 & 3925936 & 4612276 & 32.8 \\
\hline wt Replicate 2 & 45960996 & 24914782 & 54.2 & 5574458 & 6802012 & 35.2 \\
\hline nrpd1 Replicate 1 & 38517066 & 10699950 & 27.8 & 4953248 & 1839721 & 32.0 \\
\hline nrpd1 Replicate 2 & 28406775 & 12768877 & 45.0 & 5995670 & 2415118 & 35.7 \\
\hline \multicolumn{7}{|l|}{ Capsella leaves } \\
\hline wt Replicate 1 & 27409329 & 16846352 & 61.5 & 5122709 & 3597197 & 30.7 \\
\hline wt Replicate 2 & 22259993 & 14208445 & 63.8 & 4161497 & 3302984 & 32.9 \\
\hline nrpd1 Replicate 1 & 16003678 & 8919400 & 55.7 & 2748976 & 1830154 & 29.7 \\
\hline nrpd1 Replicate 2 & 38120832 & 23880822 & 62.6 & 7534940 & 4934084 & 30.2 \\
\hline
\end{tabular}

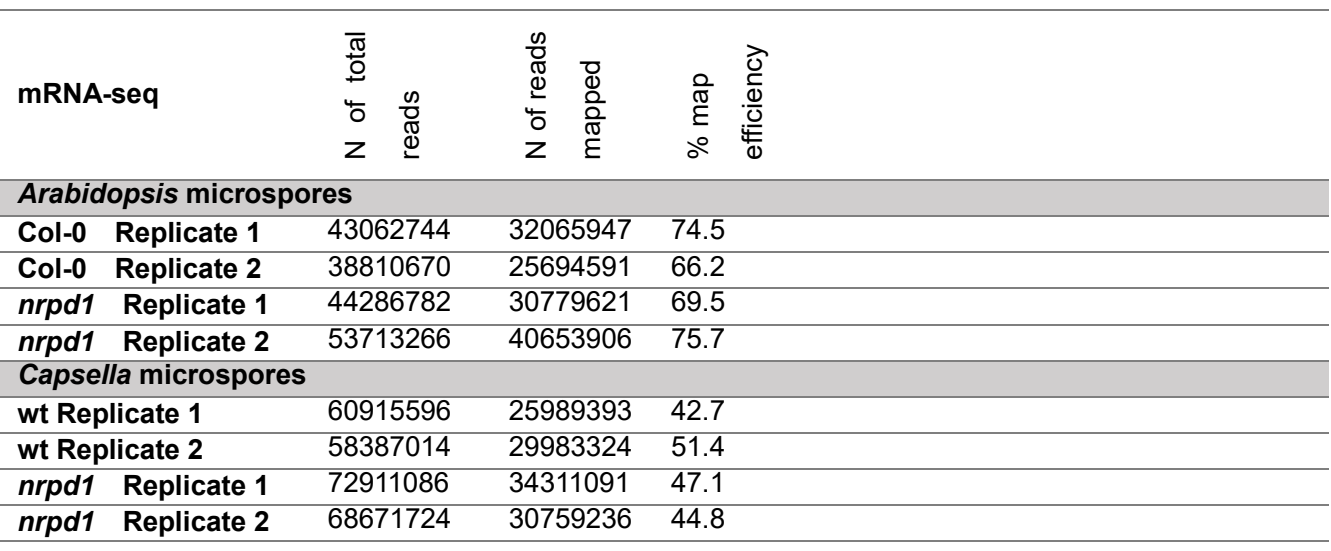

Bisulfite-seq

Capsella leaves

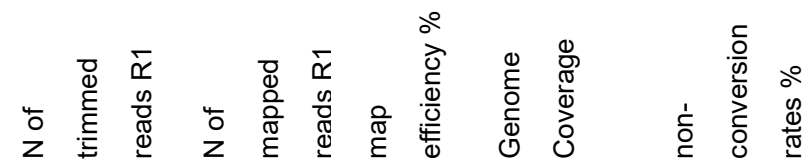

\begin{tabular}{llllllll}
\hline wt Replicate 1 & 31169177 & 11598232 & 37.2 & 5.4 & 0.5 \\
\hline wt Replicate 2 & 34936119 & 13801777 & 39.5 & 6.2 & 1.4 \\
\hline nrpd1 & Replicate 1 & 29591259 & 11329390 & 38.3 & 5.2 & 0.6 \\
\hline nrpd1 & Replicate 2 & 35716742 & 13160912 & 36.8 & 6.0 & 0.5 \\
\hline
\end{tabular}

\begin{tabular}{|c|c|c|}
\hline $\begin{array}{l}\text { WGS } \\
\text { Capsella leaves }\end{array}$ & 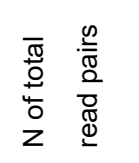 & $\begin{array}{l}\frac{1}{\overparen{\pi}} \\
\frac{\pi}{0} \\
\frac{\partial}{0}\end{array}$ \\
\hline wt & 42815402 & $68 x$ \\
\hline nrpd1_1 & 39261629 & $41 x$ \\
\hline nrpd1_2 & 33173870 & $44 x$ \\
\hline nrpd1_3 & 32911305 & $52 x$ \\
\hline nrpd1_4 & 45561371 & $65 x$ \\
\hline nrpd1_5 & 31298911 & $48 x$ \\
\hline
\end{tabular}

